\title{
MAXIMAL CLIQUE AND GRAPH COLORING HEURISTICS FOR OVERLAPPING COMMUNITY DETECTION IN NETWORK PSYCHOMETRICS
}

\begin{abstract}
Community detection is an important aspect of network psychometrics. An inherent limitation of popular methods such as the walktrap and spin glass algorithms is that they do not allow vertices (e.g., symptoms) to have membership in more than one community. Clique percolation remedies this limitation by allowing overlapping communities. However, clique percolation does not necessarily produce solutions in accordance with the standard definition of 'community' (i.e., a dense subgraph of the network), often fails to assign all vertices to at least one community, and presents formidable model selection challenges. In this paper, we present two new heuristics for overlapping community detection that are less prone to these problems. The first heuristic assembles communities using maximal cliques, whereas the second produces the communities using cliques identified from colorings of the network graph. Four psychological networks from the literature are used to demonstrate the new heuristics. The results indicate that both heuristics produce communities of dense subgraphs in accordance with the traditional definition of a community and tend to assign all (or almost all) vertices to a community.
\end{abstract}

Key words: network psychometrics, overlapping community detection, maximal cliques, graph coloring, clique percolation 


\section{Introduction}

Network psychometrics focuses on the estimation and analysis of network models for psychological items such as symptoms or conditions. The vertices of the networks are the items. The edges of the network reflect connections between items, and their weights (i.e., the strength of the association between two items) are commonly estimated from binary or Likert scale data using statistical methods. For binary data, an $l_{1}$-regularized logistic regression approach based on the inverse Ising model has commonly been used (van Borkulo et al., 2014). For Likert scale data, non-regularized partial correlation (Williams \& Rast, 2020; Williams et al., 2019) and $l_{1^{-}}$ regularized Gaussian graphical models (Friedman et al., 2008, 2014) are widely implemented. Our focus herein is not on the estimation process, but on detecting communities of the psychological items (vertices) based on their estimated edges. We assume an undirected network with positive edge weights, which is common in the literature (Epskamp \& Fried, 2018).

Various methods have been used to identify communities of vertices based on the edge weights. If a partition of the vertices is desirable, then many approaches are possible including hierarchical clustering (Baker \& Hubert, 1976), spectral clustering (von Luxburg, 2007), the walktrap algorithm (Pons \& Latapy, 2005), and the spin-glass algorithm (Reichardt \& Bornholdt, 2006). Comparisons of some of these methods have been reported by Gates et al. (2016) and Hoffman et al. (2018). The walktrap and spin-glass algorithms have been especially popular in the recent network psychometrics literature (Briganti et al., 2018; Choi et al., 2017; Christensen, 2018; Fried, 2016; Golino \& Demetriou, 2017; Golino \& Epskamp, 2017; Hevey, 2018; Jones et al. 2018; Kendler et al., 2018; McElroy \& Patalay, 2019).

One of the major limitations of the aforementioned procedures is that they produce partitions, which do not permit vertices to have membership in more than one community. 
Clique percolation (Palla et al., 2005) is a method that overcomes this limitation by allowing overlapping communities. Essentially, clique percolation begins with the identification of all possible cliques (i.e., complete subgraphs) of $k$ vertices. Next, the vertices are assembled into communities based on the formation of chains of adjacent cliques that share $k-1$ vertices. In the case of weighted networks, cliques may be filtered based on their intensity, which is measured as the geometric mean of their edge weights (Farkas et al., 2007). In recent years, clique percolation has received significant attention in network psychometrics applications (Blanken et al., 2018; Cosgrove et al., 2021; Kaiser et al., 2021; Lange, 2019; Lange \& Zickfeld, 2021).

Cliques have long been recognized in the psychometric literature for their practicality in the discovery of structure in psychological networks (Hubert, 1974a; Luce, 1950; Peay, 1975), and we support their recent use for the detection of overlapping communities. However, we also believe that there are opportunities to strengthen the role of cliques in the analysis of psychological networks beyond the specific method of clique percolation. In part, these opportunities stem from well-recognized limitations of clique percolation. Perhaps the most serious limitation is that clique percolation does not actually seek communities in the traditional sense of dense subgraphs ${ }^{1}$, but rather subgraphs that contain cliques that are chained together based on the aforementioned definition of adjacency (i.e., sharing $k-1$ vertices). As explained by Fortunato (2010, p. 131): “A more fundamental issue is the fact that the method does not look for actual communities, consistent with the shared notion of dense subgraphs, but for subgraphs 'containing' many cliques, which may be quite different objects than communities (for instance, they could be 'chains' of cliques with low internal edge density)." Two other drawbacks of clique percolation observed by Fortunato (2010) are the somewhat ad hoc selection of the clique

\footnotetext{
${ }^{1}$ The density of a graph (or subgraph) is the number of edges in the graph divided by the number of possible edges. If there are $n$ vertices in the graph (or subgraph), then $(n(n-1)) / 2$ is the number of possible edges.
} 
size and intensity threshold, as well as the potential for a large number of vertices to lack membership in any community. Yet another concern, which is especially relevant here, is that psychological networks tend to be much smaller (i.e., have far fewer vertices) and denser (i.e., have a greater proportion of edges between all possible pairs of vertices) than the networks for which clique percolation was originally designed.

We have developed two new heuristics for overlapping community detection that overcome some of limitations of clique percolation. The first, MCHOC - Maximal Clique Heuristic for Overlapping Communities, uses all maximal cliques of a size exceeding some user specified threshold. The second, GCHOC - Graph Coloring Heuristic for Overlapping Communities, is similar but uses only those cliques obtained from a coloring of the network graph. ${ }^{2}$ Both heuristics emphasize the use of the largest cliques in assembling communities for psychological networks. Accordingly, the heuristics tend to identify a small group of chained cliques that produce very dense communities, thus addressing the major limitation of clique percolation observed by Fortunato (2010).

Our heuristics were also designed with the recognition that a clique's intensity is not the only measure of its contribution to the network. For example, clique centrality could also be considered (Vogiatzis et al., 2015). Whereas intensity measures the internal cohesion of a clique, clique centrality measures the extent to which a clique serves as a bridge to other vertices in the network, and it is well-recognized that this type of 'bridging' in networks is also important (Jones et al., 2019). The fact that our heuristics do not require the specification of a somewhat arbitrary threshold for intensity overcomes one of the other limitations of clique percolation noted by Fortunato (2010). Finally, our heuristics are designed such that, if a vertex is a member

\footnotetext{
${ }^{2}$ Cliques and graph coloring are closely related. Specifically, an important version of the graph coloring problem can be viewed as finding the minimum number of cliques necessary to cover all of the vertices in the network graph.
} 
of at least one clique in the list of cliques, then it will be assigned to a community. This enables the researcher to circumvent another potential limitation of clique percolation; namely, a large number of unassigned vertices.

Section 2 begins with formal definitions of relevant terms, such as cliques, $k$-cliques, maximal cliques, maximum cliques, intensity, clique centrality, and $p$-coloring. This is followed by a presentation of mathematical programming models for finding maximal and maximum cliques, cliques that maximize centrality, and $p$-colorings of a graph, as well as a precise description of the new heuristic methods (MCHOC and GCHOC) for finding overlapping communities. Sections 3, 4, 5, and 6 demonstrate applications of the methods to four published psychological networks from the clinical literature (Dalege et al., 2017; Kessler et al., 2004; Kendler et al., 2018; Lange \& Zickfeld, 2021). The paper concludes in Section 7 with a summary and discussion of limitations and extensions.

\section{Models and Methods}

\subsection{Definitions}

We consider psychological networks that consist of a set of $n$ vertices, $V=\{1,2, \ldots, n\}$, that represent the items (e.g., symptoms) under study. The network graph, $G(V, E)$, is defined by $V$ and a set of undirected edges, $E=\{i, j\}$, between pairs of vertices. For notational convenience, the ordering of the vertices in an edge is arbitrary and $\{i, j\}$ is considered the same $\{j, i\}$. A clique, $C \subseteq V$, is a set of vertices that form a complete subgraph of $G(V, E)$ consisting of the vertices in $C$. Specifically, for any pair of vertices in $C$, there exists an undirected edge between those vertices or, more formally, $\{i, j\} \in C \rightarrow\{i, j\} \in E$. A $k$-clique, $C_{k}$, is a clique consisting of

exactly $k$ vertices. That is, the number of vertices in $C_{k}$, which is denoted as $\left|C_{k}\right|$ and represents the size of the clique, is equal to $k$. Two $k$-cliques, $C_{k}^{1}$ and $C_{k}^{2}$ are said to be $k$-1 adjacent if they 
share $k-1$ common vertices. ${ }^{3}$ A maximal set of $k-1$ adjacent cliques forms a community or module when considered within the framework of the clique percolation method (Adamcsek et al., 2006; Palla et al., 2005).

A $k$-clique, $C_{k}$, is said to be maximal if no vertices can be added to $C_{k}$ so as to produce a larger clique. If $C_{k}$ is maximal, then it consists of $k$ smaller cliques of size $k-1$ that can be obtained via deletion of exactly one vertex in $C_{k}$. However, none of these smaller cliques of size $k-1$ is maximal because the larger clique of size $k$ could be realized by adding back the deleted vertex. By contrast, $C_{k}$ is a maximum clique if there is no larger clique in the network graph. This distinction between maximal and maximum cliques is important and can be summarized concisely by the statement that a maximum clique is a largest maximal clique in the network graph. Many graphs have multiple maximum cliques.

As described by Vogiatzis et al. (2015), the degree centrality of a $k$-clique $\left(C_{k}\right), D\left(C_{k}\right)$, is the number of vertices that are not members of $C_{k}$, yet are connected by an edge to at least onemember of $C_{k}$. In this respect, the degree centrality of a clique can be perceived as a measure of the extent to which it serves as a bridge to other vertices in the network.

In the case of a weighted network, where nonnegative edge weights $a_{i j}$ are available for each edge $\{i, j\} \in E$, it is possible to compute the intensity of a $k$-clique as the geometric mean of the edge weights associated with the clique as follows:

$$
I\left(C_{k}\right)=\left(\prod_{\{i, j\} \in C_{k}} a_{i j}\right)^{2 /(k(k-1))}
$$

Intensity thresholds have been used to determine the inclusion or exclusion of a clique within the framework of clique percolation for weighted graphs (Farkas et al., 2007).

\footnotetext{
${ }^{3}$ Two cliques, $C_{l}^{1}$ and $C_{k}^{2}$ where $l>k$ are also said to be $k$ - 1 adjacent if they share $k-1$ common vertices
} 
A $p$-coloring of a network graph pertains to the assignment of one of $p$ colors to each vertex of the graph, such that an edge exists between every pair of vertices that have the same color. More formally, if we define $b(i)$ as the color of vertex $i$, then $(b(i)=b(j)) \rightarrow\{i, j\} \in E$. An assignment of $p$ colors to vertices that meets this condition is known as a $p$-coloring of $G(V, E)$. The chromatic number of the graph $G(V, E)$ is the minimum value of $p$ that allows for a $p$ coloring of $G(V, E)$. Because vertices with the same color must be connected by an edge, each subset of vertices of the same color is a clique.

\subsection{Maximum Cliques}

Pioneering work on the development of enumerative algorithms for finding all the cliques of a graph was undertaken by Harary and Ross (1957). The problem of finding a maximum clique of an arbitrary graph is simpler, but known to be NP-hard (Garey \& Johnson, 1979; Pardalos \& Xue, 1994). For several decades, branch-and-bound programming has been successfully employed to find the maximum clique of graphs of modest size (Tarjan \& Trojanowski, 1977). The maximum clique problem can also be formulated as an integer linear programming model. The simplest formulation uses decision variables $x_{i}=1$ if vertex $i$ is a member of the maximum clique and $x_{i}=0$ otherwise, for all $i \in V$. The objective function is to maximize the sum of these binary variables, which represents the total number of vertices in the clique:

$$
\text { Maximize: } Z_{\mathrm{MC}}=\sum_{i \in V} x_{i}
$$

The primary constraint set requires that, for any pair of vertices not connected by an edge, no more than one of the two vertices in the pair can be a member of the clique:

$$
x_{i}+x_{j} \leq 1 \quad \forall\{i, j\} \notin E .
$$

Finally, there is a constraint set to assure that the decision variables are binary:

$$
x_{i} \in\{0,1\} \quad \forall i \in V .
$$


It is well known that the integer programming formulation associated with Equations (2)-(4) is not sharp (Bomze et al., 1999; Pardalos \& Xue, 1994). That is, the solution to the linear programming (LP) relaxation (i.e., the linear program realized by replacement of the binary restrictions on the variables in Equation (4) with the constraints $x_{i} \geq 0$ for all $i \in V$ ) of the formulation often has a large number of fractional variables and the objective function value of the LP-relaxation is commonly much larger than the actual size of the maximum clique. Nevertheless, the model performs satisfactorily for smaller networks (e.g., $n<100)$.

When the formulation associated with Equations (2)-(4) is solved, the vertices associated with the maximum clique, $C$, are identified by the binary decision variables that assume a value of one, that is, $i \in C: x_{i}=1$. However, the maximum clique obtained is frequently not unique. That is, there are often other maximum cliques in the graph. To determine if there is another maximum clique, the formulation can be resolved after appending the following constraint, which assures that the same maximum clique is not found again:

$$
\sum_{i \in V} x_{i} \leq|C|-1
$$

By iteratively appending constraints of type (5) and resolving, all of the maximum cliques can be efficiently obtained. Moreover, by continuing to append such constraints, it is possible to extract all maximal $k$-cliques of a cardinality that exceeds a user-prescribed threshold. This is useful when there are only a few maximum cliques (e.g., a few 5-cliques) and the researcher would also like to extract all maximal 4-cliques and 3-cliques. We have developed a MATLAB (MATLAB, 2020) m-file, ilp_maxclique.m that allows for extraction of all of the maximum cliques equal to or greater than a user-specified size. The program also computes the intensity and clique degree centrality of each of the extracted cliques. The intensity of a clique is important because it 
measures the strength of its internal cohesion, whereas clique degree centrality provides information regarding the reach of the clique to other vertices in the network.

\subsection{Clique Centrality}

Vogiatzis et al. (2015) presented a suite of integer programming models that can be used to extract the most central (or least central) cliques within a network. They presented models based on degree, closeness, and betweenness centrality measures. Our focus here is on degree centrality and the identification of the most central clique in the network. The degree centrality of a clique $C$ is defined as the number of vertices that are not members of $C$, yet are connected by at least one edge to at least one of the members of $C$. The integer program for finding the most central clique requires an additional set of decision variables: $y_{i}=1$ if vertex $i$ is not a member of the clique, yet is connected to at least one member of the clique. The objective function is to maximize the sum of the $y_{i}$ variables, which represents the degree centrality of the clique:

$$
\text { Maximize: } Z_{\mathrm{DC}}=\sum_{i \in V} y_{i}
$$

A constraint set is necessary to prohibit a vertex from being both a member of the clique and an external vertex connected by an edge to one or more (but not all) of the members of the clique:

$$
x_{i}+y_{i} \leq 1 \quad \forall i \in V .
$$

Another constraint set is necessary is required to assure that each $y_{i}$ variable cannot assume a value of one unless vertex $i$ is connected to one or more members of the clique:

$$
y_{i}-\sum_{j:\{i, j\} \in E} x_{j} \leq 0 \quad \forall i \in V .
$$

Constraint sets (3) and (4) are also required, as are binary restrictions on the $y_{i}$ variables:

$$
y_{i} \in\{0,1\} \quad \forall i \in V .
$$

We have developed a MATLAB m-file, ilp_centrality.m, that allows for identification of a clique with maximum degree centrality. 


\subsection{Graph Coloring}

Graph-coloring has a long and rich history in the mathematical sciences. May (1965) and Dailey (1978) observed that a coloring problem was originally brought to the attention of Augustus de Morgan by Francis Guthrie in the mid-1800s (Guthrie, 1880). The problem, commonly known as the four-color map problem, focused on the coloring of a (planar) map of the counties of England and it was conjectured that no more than four colors were required to color the map such that no two adjacent counties were of the same color. Early attempts at proving the conjecture (Kempe, 1879; Tait, 1880a, b) were subsequently shown to be invalid, and an acceptable proof was not provided until the 1970s (Appel \& Haken, 1977a, b). Up through the mid- $20^{\text {th }}$ century, most of the attention on coloring problems was restricted to the four-color map problem. However, the pioneering work of Brooks (1941) initiated a broader conceptualization of the problem that focused on the coloring of the vertices of a graph.

Subsequently, there has been a considerable interest with respect to both the development of methods for graph coloring and the applicability of the problem to various scientific disciplines.

In the field of quantitative psychology, Hubert (1974a, 1974b) was one of the earliest proponents of graph coloring. He recognized the close relationship between assigning color labels and assigning cluster memberships to objects, and noted that the implications of this relationship were "awesome" in light of the vast body of literature on graph coloring (Hubert, 1974a, p. 297). As recently noted by Brusco et al. (2021), graph coloring has direct relevance to important clustering methodologies such as complete-linkage hierarchical clustering (see, for example, Baker \& Hubert, 1976) and minimum-diameter partitioning (see, for example, Brusco, 2003; Brusco \& Steinley, 2014; Hansen \& Delattre, 1978). 
The importance of graph coloring as an approach for studying psychological network relationships is also based on the recent work of Marsman et al. (2018) and Savi et al. (2019), who have acknowledged the linkage between Ising network models and the work of Fortuin and Kasteleyn (1972) pertaining to the random-cluster model. The random-cluster model forms a unifying framework for the Ising model (Ising, 1925), the closely-related percolation model (Broadbent \& Hammersley, 1957), and Potts networks (Ashkin \& Teller, 1943; Potts, 1952). The close relationship between the ground state of the Potts model and the coloring of the nodes of a graph forms a basis for the relationship between graph coloring and one of the most popular network models in the literature, the Ising network model (Kruis \& Maris, 2016; Marsman et al., 2015). A direct Ising spin formulation of the graph-coloring problem is also possible (Brusco et al., 2021; Lucas, 2014).

An integer linear programming formulation for finding the chromatic number and producing a $p$-coloring uses decision variables: (i) $u_{i q}=1$ if vertex $i$ is assigned color $q$ and $u_{i q}=$ 0 otherwise, for all $i \in V$ and $1 \leq q \leq p$, and (ii) $w_{q}=1$ if color $q$ is used and $w_{q}=0$ otherwise, for all $1 \leq q \leq p$. The objective is to minimize the number of colors used:

$$
\text { Minimize: } Z_{\mathrm{GC}}=\sum_{q=1}^{p} w_{q}
$$

The first constraint set (11) requires the assignment of exactly one color to each vertex.

$$
\sum_{q=1}^{p} u_{i q}=1 \quad \forall i \in V ;
$$

The second constraint set (12) assures that, for each pair of vertices that are not connected by an edge (i.e., $\{i, j\} \notin E$ ) and each color $q(1 \leq q \leq p)$, no more than one of the two vertices in the pair can be assigned color $q$.

$$
u_{i q}+u_{j q} \leq w_{k} \quad \forall\{i, j\} \notin E \text { and } 1 \leq q \leq p,
$$


Constraints (13) and (14) are included to tighten the formulation by prohibiting alternative optimal solutions that differ only in color labeling (Méndez-Díaz \& Zabala, 2006, 2008).

$$
\begin{array}{cl}
w_{q} \leq \sum_{i \in V} u_{i q} & \forall 1 \leq q \leq p, \\
w_{q} \leq w_{q}-1 & \forall 2 \leq q \leq p,
\end{array}
$$

Binary restrictions on the decision variables are imposed in constraints (15) and (16).

$$
\begin{array}{ll}
u_{i q} \in\{0,1\} & \forall i \in V \text { and } 1 \leq q \leq p, \\
w_{q} \in\{0,1\} & 1 \leq q \leq p .
\end{array}
$$

A solution to the model posed by Equations (10)-(16) will find a coloring using the minimum number (the chromatic number) of colors. If the problem is infeasible, then more than $p$ colors are necessary to color the graph. We developed the MATLAB m-file ilp_graph_coloring.m for solving the problem posed by Equations (10)-(16). Many psychological networks have a large number of possible $p$-colorings. Although the integer programming model could be modified to identify all colorings in a manner similar to that described for ilp_maxclique.m in Section 2.2, such an approach is computationally demanding and unnecessary. A preferable approach is discussed in the next section.

\subsection{Maximal Clique and Graph Coloring Heuristics for Finding Overlapping Communities}

We strongly support the emphasis on cliques for discovering structure in psychological networks. However, as noted in Section 1, Fortunato (2010) has identified several potential drawbacks of clique percolation and psychological networks tend to be much smaller and much denser than the networks for which the method was originally designed. In light of these limitations, we propose the following principles for clique-based analysis when studying psychological networks: (1) Focus on maximal cliques, (2) Focus on maximum (or, at least, near-maximum) cliques, (3) Clique intensity should be deemphasized in the construction 
communities because other characteristics of a clique (e.g., centrality) are also important, (4) In accordance with clique percolation, $k$-1 adjacency is effective for aggregating cliques into communities, and (5) If a finer solution with more communities of greater density is desired, then consider using graph coloring to reduce the number of cliques.

These principles served as the foundation for our development of the MCHOC and GCHOC heuristics, which are implemented in the MATLAB m-files mchoc.m and gchoc.m, respectively. The heuristics differ only with respect to how the candidate cliques for assembling the communities are generated. In MCHOC, all maximal cliques of size $k_{\min }$ or larger are obtained, whereas in GCHOC, only those cliques of size $k_{\min }$ or larger that are part of a $p$ coloring of the network graph are candidates. The pseudocode of the basic structure for both heuristics is provided in Figure 1. The inputs to the algorithms are the (nonnegative) adjacency matrix for the network graph, $G(V, E)$ and minimum clique size $\left(k_{\min }\right) .{ }^{4}$ The default setting is $k_{\min }$ $=3$, as 2-cliques are merely edges and are commonly of no practical interest. The algorithm description in Figure 1 uses several notations heretofore undefined: (i) $c$ is the number of cliques extracted; (ii) $T$ is the number of communities, which is initialized to zero, (iii) $\chi(d)$ indicates the community to which clique $d$ is assigned and, initially, $\chi(d)=0$ for $1 \leq d \leq c$, and (iv) $H_{t}$, which does not exist at the beginning of the heuristic because there are no communities, will eventually contain the vertices that are assigned to each community $t$ (for $1 \leq t \leq T$ ).

\section{[Insert Figure 1 About Here]}

The first step is to generate the candidate cliques. The algorithms used for generating all maximal cliques (used in $\mathrm{MCHOC}$ ) and all p-colorings (used to obtain the cliques for $\mathrm{GCHOC}$ ) of the graph are similar; however, the latter problem is much more computationally demanding.

\footnotetext{
${ }^{4}$ Also, the number of colors, $p$, for GCHOC should typically be set to the chromatic number for the network graph.
} 
We adopted an implicit enumeration algorithm (Brusco \& Stahl, 2005, Ch. 3) for finding all $p$ colorings of a graph, and this algorithm is incorporated in gchoc.m. A formal description of the algorithm is provided in the Appendix. Some modifications of the algorithm for a particular case of $p=2$, where vertices colored $b(i)=1$ are in the clique and those colored $b(i)=2$ are not in the clique, facilitate an adaptation for finding all maximal cliques and this modified version is incorporated in mchoc.m. This resulting algorithm is much faster than using the integer programming approach described in Section 2.2.

The outer loop of the assignment algorithm controls the clique size, which begins at the maximum $\left(k_{\max }\right)$ and is decremented by 1 until the lower limit of $k_{\min }$. The 'while' loop controlled by flag1 assures that the larger cliques will be exhaustively evaluated for assignment before cliques of smaller size are assessed. Two types of clique assignment are possible. The first type of assignment, controlled by flag2, pertains to assigning cliques to existing communities. If a clique, $C^{d}$, is to be assigned to an existing community, then it must meet three conditions: (i) it must be of the current clique size (i.e. $\left|C^{d}\right|=k$ ), (ii) it must be $k$-1 adjacent to a clique, $d^{\prime}$, that is an existing member of the community (i.e., $\left|C^{d} \cap C^{d^{\prime}}\right|=\left|C^{d}\right|-1$ ), and (iii) it must contribute at least one vertex that has not previously been assigned to a community (i.e., $\bar{V} \cap C^{d} \neq \emptyset$, where $\bar{V}=V \backslash\left\{\bigcup_{t=1}^{T} H_{t}\right\}$ is the set of unassigned vertices). The second type of assignment, controlled by flag 3 , is the creation of a new community. This type of evaluation, which is only conducted after it is no longer possible to find a clique of size $k$ to assign to an existing community, has only two of the three conditions as requirements: current clique size and contribution of at least one vertex not previously assigned.

The algorithm proceeds to add the cliques to communities based on $k$ - 1 adjacency, beginning with the largest cliques first, until one of two possible termination conditions is 
satisfied: all vertices have been assigned to a community, or no more cliques can be assigned to a community. Although they do not require parameter specification other than the selection of $k_{\min }$, which can be trivially set to its default value of $k_{\min }=3$, the MCHOC and $\mathrm{GCHOC}$ heuristics are sensitive to the ordering of the cliques and, in this respect, are similar to some hierarchical clustering procedures (van der Kloot et al., 2005). Consistent with the approach used by van der Kloot et al., we recommend running the heuristics multiple times using different random permutations of the list of cliques. As we demonstrate in later sections, the selection of an overlapping community solution can be based on relative community sizes and densities. A smaller number of communities is preferable if it does not result in very large communities of low density. For a given number of communities, a simple rule would be to select the solution that maximizes the minimum (across all communities) density.

\section{Example 1: Obama Network}

\subsection{Data}

We first consider a small network pertaining to $n=10$ perceived attitudes toward descriptive attributes of Barack Obama: 1 (Moral), 2 (Leadership), 3 (Caring), 4 (Knowledgeable), 5 (Intelligent), 6 (Honest), 7 (Angry), 8 (Hopeful), 9 (Afraid), and 10 (Proud). The data were collected during the 2012 U.S. Presidential Election cycle. Dalege et al. (2017) estimated the network using the IsingFit model for binary data, as described by van Borkulo et al. (2014). Only a few small negative edges were present in the estimated network and Dalege et al. (2017) focused on the absolute value of the edge weights in their community detection algorithm. Here, we use the absolute value of all edge weights exceeding 0.25 in absolute value.

\subsection{Analysis}


3.2.1. MCHOC Results. We used the mchoc . m program to find all maximal cliques of size $k=3$ or larger. This resulted in the identification of two maximal 7-cliques, $C_{7}^{1}=\{1,2,3,4,5,6$, $10\}$ and $C_{7}^{2}=\{2,3,4,5,6,8,10\}$, as well as one maximal 6-clique $C_{6}^{3}=\{2,3,6,7,8,9\}$ and one maximal 5-clique $C_{5}^{4}=\{1,2,3,6,9\}$. The intensities of these three cliques are $.7670, .7005$, .6324 , and .7511 , respectively. The clique degree centralities are $3,3,4$, and 5 , respectively. Because of the high density of this network, the clique degree centralities are not especially interesting. That is, for each of the three cliques, all of the vertices not in the clique share an edge with one or more vertices in the clique.

For the Obama network, the communities obtained by MCHOC are unaffected by the ordering of the cliques. The two maximal 7-cliques, $C_{7}^{1}$ and $C_{7}^{2}$, are 6-adjacent and, therefore, form a community. These two cliques are shown with solid boundaries in Figure 2 and the resulting community, Community 1 , is shaded in yellow. Community 1 comprised eight of the 10 descriptive attributes, with only the two negative attributes $\{7$ : Angry, 9: Afraid $\}$ excluded from the community. The maximum 6-clique, $C_{6}^{3}$, is not 5-adjacent with either of the 7-cliques and, therefore, forms its own community (Community 2) shaded in blue in Figure 2. Community 2 does contain $\{7$ : Angry, 9: Afraid $\}$, as well as four other descriptive attributes that have membership in both communities \{2: Leadership, 3: Caring, 6: Honest, 8: Hopeful $\}$. The overlap of the two communities is shaded green in Figure 2. The yellow portion represents Community 1, which corresponds to leadership; the blue portion represents Community 2, which corresponds to negative attributes/emotions; and the green portion comprises the overlap between the two communities, which largely reflects positive attributes/emotions. Because the first two communities jointly contain all 10 characteristics, our procedure terminates in Step 3 with $k=6$. 
3.2.2. GCHOC Results. The gchoc_gc.m algorithm was used to find all of the 2-colorings of the Obama network graph. Eight 2-colorings were found. They are displayed in Table 1 along with the average intensity of the cliques associated with the 2-coloring. It is interesting to observe that $C_{7}^{1}$ and $C_{6}^{3}$ are both associated with a 2-coloring; however, $C_{7}^{2}$ is not. The reason is that the characteristics that are not members of $C_{7}^{2}$ are $\{1$ : Moral, 7: Angry, 9: Afraid $\}$, and these three characteristics do not form a 3-clique because there is no edge connecting Moral and Angry. In addition to the 2-colorings associated with $C_{7}^{1}$ and $C_{6}^{3}$, there are three 2-colorings associated with non-maximal 6-cliques and four-cliques, as well as three 2-colorings associated with a pair of 5-cliques. Therefore, the use of GCHOC instead of MCHOC, which restricts consideration to only 7-cliques and 6-cliques that are components of a 2-coloring, would result in \{8: hopeful\} being dropped from Community 1.

[Insert Table 1 About Here]

4. Example 2: NCS-R Network

\subsection{Data}

Next, we analyze a network pertaining to $n=18$ major depressive and generalized anxiety symptoms, which was obtained via application of the IsingFit procedure (van Borkulo et al., 2014) to data originally collected for the National Comorbidity Study - Replication (NCS-R:

Kessler et al., 2004). This network has been analyzed several times in the literature (Borsboom et al., 2017; Brusco et al., 2019; Forbes et al., 2017; Steinley et al., 2017). Like the previous example, there were only a few small negative edge weights and the absolute value of these edge weights was used in the construction of the network.

\subsection{Analysis}


4.2.1. MCHOC Results. We applied the mchoc . m program to the NCS-R network and found 16 maximal cliques ranging in size from $k=3$ to the maximum clique size of $k=7$. These cliques, along with their intensities and clique centralities are displayed in Table 2. One interesting result is that the largest cliques in the network do not have the greatest centrality, which is consistent with the findings of Vogiatzis et al. (2015). For example, cliques $C_{7}^{1}$ and $C_{7}^{5}$ are maximum 7-cliques with clique degree centralities of $D\left(C_{7}^{1}\right)=D\left(C_{7}^{5}\right)=9$, which means they reach 9 of the 11 symptoms that are not in their respective cliques. By contrast, $C_{4}^{9}, C_{4}^{12}, C_{4}^{14}$, and $C_{4}^{15}$ are only 4-cliques; however, their clique degree centralities of $D\left(C_{4}^{9}\right)=D\left(C_{4}^{12}\right)=D\left(C_{4}^{14}\right)=$ $D\left(C_{4}^{15}\right)=14$ means that they reach all 14 symptoms not in their clique.

\section{[Insert Table 2 About Here]}

Like the Obama network, the communities produced by MCHOC were not influenced by the ordering of the cliques. There are five maximum cliques (the 7 -cliques, $C_{7}^{1}-C_{7}^{5}$ ) in Table 2 that produce two non-overlapping communities. Cliques $C_{7}^{1}, C_{7}^{2}$, and $C_{7}^{3}$ are 6-adjacent and produce Community 1, which consists of all nine depression symptoms \{1: Depressed mood, 2: Loss of interest, 3: Weight problems, 4: Sleep problems, 5: Psychomotor disturbances, 6: Fatigue, 7: Self-reproach, 8: Concentration problems, 9: Suicidal ideation $\}$. Cliques $C_{7}^{4}$ and $C_{7}^{5}$ are 6adjacent and produce Community 2 , which consists of eight of the nine anxiety symptoms $\{11$ : Anxiety about >1 event. 12: No control over anxiety, 13: Feeling on edge, 14: Fatigue, 15: Concentration problems, 16: Irritability, 17: Muscle tension, 18: Sleep problems \}. The remaining symptom $\{10$ : Chronic anxiety/worry $\}$ joins Community 1 because of the 4 -cliques $C_{4}^{10}=\{1,5$, $7,10\}$ and $C_{4}^{11}=\{1,7,9,10\}$. The community structure is summarized via a permuted network matrix in Figure 3. 
Community 1 is shaded in yellow and Community 2 is shaded in blue in Figure 3. Solid borders are used to highlight three of the 7-cliques and dashed borders indicate one of the 4cliques that joins symptom $\{10$ : Chronic anxiety/worry $\}$ to Community 1 . The two communities are non-overlapping and very dense. One aspect of the solution that might be disconcerting is that symptoms 10 and 11 have a very strong edge weight but are in different communities because there is no third symptom for which both 10 and 11 share an edge so as to form a 3clique. This is an artifact of the skip-out structure of the NCS-R questionnaire, whereby question 11 is only answered if question 10 is answered affirmatively.

4.2.2. GCHOC Results. The gchoc_gc.m algorithm was used to determine the chromatic number of four and to find all of the 4-colorings of the NCS-R network graph. Forty 4-colorings were found. Replacing MCHOC with GCHOC and using only cliques that were present in the 4colorings of the network graph would result in a rather different community structure. Only two of the maximum 7-cliques were present in some of the 4-colorings: $C_{7}^{3}=\{1,2,3,4,6,7,8\}$ and $C_{7}^{5}=\{11,12,13,14,15,16,18\}$. These two cliques would define Communities 1 and 2, respectively. Symptoms 5 and 17 were assigned the same color in all 40 colorings, and symptoms 9 and 10 also shared the same color in all 40 colorings. The largest clique in which symptoms 5 and 17 appeared was the maximal 5-clique $C_{5}^{6}=\{5,14,15,16,17\}$. Because this clique is not 4-adjacent to either $C_{7}^{3}$ or $C_{7}^{5}$, it formed its own third community (Community 3 ). Likewise, the largest clique in which symptoms 9 and 10 appear is the maximal 4-clique $C_{4}^{11}=$ $\{1,7,9,10\}$ and, because this clique is not 3 -adjacent to $C_{7}^{3}, C_{7}^{5}$, or $C_{5}^{6}$, it also forms its own community (Community 4).

\section{Example 3: HAN Depression Network}

\subsection{Data}


Kendler and colleagues (2018) conducted a network analysis of $n=19$ depression symptoms from DSM-IV and elsewhere in Han Chinese women. In this application, the raw data measurements were binary and pertained to the endorsement or non-endorsement of the symptoms by each respondent. Like Examples 1 and 2, the regularized logistic regression approach for fitting the Ising model (van Borkulo et al., 2014) was used to estimate the edgeweight matrix for the data. Kendler et al. (2018) published their edge-weight values as supplemental material and we assembled them into an $n \times n$ matrix. All of the edge weights are positive and range in value from .13 to 1.71 .

\subsection{Analysis}

5.2.1. MCHOC Results. We applied the mchoc . m program to the Han depression network to generate all maximal cliques of size $k \geq 3$. The maximum clique size was only $k=5$ and there were twelve unique 5-cliques. Ten additional maximal cliques of sizes $k=4$ or $k=3$ were also identified. Table 3 provides all 22 cliques, along with their intensity and clique centrality measures. One of the most striking aspects of Table 3 is that Clique $C_{5}^{1}=\{6,9,10,16,18\}$, has, by far, the largest intensity, $I\left(C_{5}^{1}\right)=.6665$, but has the smallest clique degree centrality, $D\left(C_{5}^{1}\right)=$ 9. Contrastingly, Clique $C_{5}^{4}=\{4,9,13,18,19\}$, has the smallest intensity, $I\left(C_{5}^{4}\right)=.2269$, but is tied for the largest clique degree centrality, $D\left(C_{5}^{4}\right)=13$. These results exemplify the importance of considering both intensity and centrality when evaluating cliques. Clique $C_{5}^{1}$ is much more internally cohesive than Clique $C_{5}^{4}$, as its intensity is nearly three times greater. However, Clique $C_{5}^{4}$ has greater reach to other areas of the network because it has connections to 13 of the 14 vertices that are not members of its clique, in contrast to Clique $C_{5}^{1}$ having only 9 of 14 possible connections.

[Insert Table 3 About Here] 
Unlike the previous two examples, the communities obtained by MCHOC are affected by the ordering of the cliques. Two-community and four-community solutions were common. Table 4 provides a two-community solution in the top panel and a four-community solution in the bottom panel. The corresponding network graphs for these two solutions, which were produced using qgraph (Epskamp et al., 2012), are displayed in Figures 4 and 5, respectively. The twocommunity solution in Table 4 has one very large community (Community 1 ) that contains 16 of the 19 vertices, with the only excluded vertices being $\{9: \downarrow$ self-esteem, 15 : irritable/angry, 18 : helplessness $\}$. Community 2 of the two-community solution is the 5 -clique $\{6$ : worthlessness, 9 :

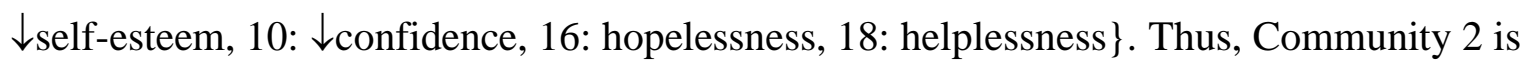
defined by the highest-intensity clique that pertains to symptoms that reflect a negative outlook (i.e., no confidence or self-esteem and feelings of worthlessness and helplessness). The overlap between the two communities corresponds to symptoms $\{6$ : worthlessness, 10: $\downarrow$ confidence, 16 : hopelessness \}, which have membership in both communities. The MCHOC algorithm terminates with $\{15$ : irritable/angry $\}$ as an isolate that is not assigned to any community. In fact, this symptom can never be assigned to a community because it is not a member of any maximal $k$ cliques of $k=3$ or larger.

[Insert Table 4 and Figures 4 and 5 About Here]

The major drawback of the two-community solution in Table 4 is the low density (.4417) of Community 1 , which is not much larger than the density of the full network (.4152). An alternative two-community solution was found where $\{17$ :crying $\}$ was placed in Community 2 rather than Community 1 . This slightly raises the density of Community 1 to .4667 (and lowers the density of Community 2 from 1.0000 to .8667); however, it is still quite low. By contrast, the four-community solution in Table 4 is far more balanced with respect to the relative sizes of the 
communities and the densities of all four communities exceed 0.7 . Community 1 of the fourcommunity solution contains the high-intensity (negative outlook) 5-clique that defined Community 2 of the two-community solution. Community 2 of the four-community solution is anchored by the 5 -clique $\{6$ : worthlessness, 8 : suicidality, 10 : $\downarrow$ confidence, 14 : unreactive mood, 16: hopelessness\}, which links suicidality to the two symptoms (hopelessness and helplessness) to which it is most strongly tied. Community 3 is largely comprised of symptoms related to the physical manifestations of depression $\{2: \Delta$ weight, appetite, $3: \Delta$ sleep, $4: \downarrow$ motor movement, 5 : fatigue, 7 : difficulty concentrating, 13 : $\downarrow$ libido $\}$. Community 4 is a 4 -clique, which corresponds to the only maximal clique in Table 3 that contains $\{11$ : distinct quality of depressed mood $\}$.

5.2.2. GCHOC Results. The chromatic number for the Han depression network graph is seven. The mchoc_gc.m algorithm was used to find all of the 7-colorings of the Han depression network graph. A total of 2124 such colorings were identified and all of the 5-cliques, 4-cliques, and 3-cliques in the 7-colorings were extracted. Only eight of the twelve 5-cliques in Table 3 were components of one or more 7-colorings. As was the case for the NCS-R example in the previous section, replacing MCHOC with GCHOC and using only the available cliques associated with a $p$-coloring results in a finer solution with more communities, which are of greater density because $k-1$ adjacency chaining is mitigated by the missing cliques. The most common solution across different permutations of the cliques is a six-community solution with densities exceeding 0.8 for each community.

The six-community solution is as follows: Community 1 (density $=.8667):\{4: \downarrow$ motor movement, 5: fatigue, 7: difficulty concentrating, 12: worse in AM, 13: $\downarrow$ libido, 19: nervousness $\}$; Community 2 (density $=.9333):\{4: \downarrow$ motor movement, 6 : worthlessness, 7 : difficulty concentrating, 10: $\downarrow$ confidence, 13: $\downarrow$ libido, 14:unreactive mood\}; Community 3 
$($ density $=.8095):\{4: \downarrow$ motor movement, 6: worthlessness, 9 : $\downarrow$ self-esteem, 10 : $\downarrow$ confidence, 16 : hopelessness, 17:crying; 18: helplessness; Community 4 (density $=.8667):\{2: \Delta$ weight, appetite, 3: $\Delta$ sleep, 4: $\downarrow$ motor movement, 5: fatigue, 8:suicidality,13: $\downarrow$ libido\}; Community 5 (density $=1.0000):\{4: \downarrow$ motor movement, 8 : suicidality, 11 : distinct quality of depressed mood, 14: unreactive mood $\}$; and $\{$ Community 6: $\{1: \downarrow$ interest, 14: unreactive mood, 16 : hopelessness\}.

\section{Example 4: Positive Emotions Network}

\subsection{Data}

For our fourth and final example, we analyze a network that is appreciably larger than the previous three, which establishes the scalability of the heuristics. Specifically, we focus on a recent example reported by Lange and Zickfeld (2021), who provided a network analysis of $n=$ 56 positive emotion statements. The statements are shown in Table 5 . The data were originally collected and factor analyzed by Weidman and Tracy (2020). The raw data are responses to 5point Likert scale statements and Lange and Zickfeld employed the Gaussian graphical modeling approach described by Epskamp and Fried (2018) to estimate the network model. Unlike the previous three examples, clique percolation was applied to the positive emotions network, which facilitates a comparison of that method to MCHOC and GCHOC.

[Insert Table 5 About Here]

\subsection{Analysis}

6.2.1. MCHOC Results. We downloaded the raw data and supplemental material provided by Lange and Zickfeld (2021) from the website: https://osf.io/b2y3x/. Next, we estimated the Gaussian graphical model using the default settings of the EBICglasso program in the ggraph R package (Epskamp et al., 2012). Subsequently, we applied the mchoc . m program to the $56 \times$ 
56 estimated network matrix to generate all maximal cliques of size $k \geq 3$. We found three maximal 7-cliques, 15 maximal 6-cliques, and 107 maximal 5-cliques. When applying MCHOC, the 7-cliques and 6 cliques were sufficient to assign most items to a community. A small number of the 5-cliques facilitates the assignment of the remainder of the items. The algorithm resulted in the identification of several solutions with ten overlapping communities, which is comparable to the nine overlapping communities plus one isolate (Ad18) obtained by Lange and Zickfeld (2021) using clique percolation. Table 6 provides a comparison of one of the 10-community solutions obtained by MCHOC with the nine-community solution of Lange and Zickfeld (2021), including the items assigned to each community and the density of each community.

\section{[Insert Table 6 About Here]}

In the supplemental material (Study2_OtherAppreciatingEmotions.R) associated with their article, Lange and Zickfeld (2021) observed that the choices of clique size and intensity threshold for clique percolation were "rather difficult", which is congruent with the arbitrariness of these parameters noted earlier. An examination of the clique percolation solution reveals that the densities of most of the communities are modest, which is consistent with the limitation noted by Fortunato (2010). Only four of the nine communities have a density that exceeds 0.80 and three of communities have densities less than 0.6. Moreover, the four communities with densities exceeding 0.80 are very small. Two of these communities are 4-cliques, one is a 5clique, and one is a five-item community that is not a 5-clique. The 4-clique consisting of gratitude items that defines Community 4 is not a maximal 4-clique. These four items are part of a maximal 6-clique that includes Ad14 and G10.

An examination of the 10-community solution obtained using MCHOC leads to the identification of much stronger community densities. Nine of the 10 communities have a density 
that exceeds 0.8 . The largest community has a density of only .5956; however, this is larger than the densities of three of the communities in the clique percolation solution. Unlike the clique percolation solution reported by Lange and Zickfeld (2021), which has two communities that are 4-cliques and one isolate, the solution obtained via MCHOC has no four-item communities or isolates. Instead, the solution MCHOC solution has two 6-clique communities (Community 5 and Community 8). The other 10-community solutions identified by MCHOC from different permutations of the maximal cliques were very similar to the one reported in Table 6. One of these removes Aw10 from Community 2, which improves the density of that community from .5956 to .6250 . However, this also affects the densities of some of the other communities, resulting in a second community with a density below 0.7 .

6.2.2. GCHOC Results. The chromatic number for the positive emotions network graph is 12. The gchoc_gc.m algorithm was used to find all of the 12-colorings of the positive emotions network graph. A total of 1329 such colorings were identified and all of the 7-cliques, 6-cliques, and 5-cliques in the 12-colorings were extracted and used to assemble communities. Once again, replacing MCHOC with GCHOC and using only those cliques appearing in a coloring resulted in a finer solution with more communities of greater density. A common solution was an 11-community solution with densities of at least 0.7424 for each community. It would seem that increasing the number of communities from 10 to 11 would be well worth the increase in the minimum community density from .6250 to .7424 .

The 11-community solution is as follows: Community 1 (density $=.7424)$ : $\{\operatorname{Ad} 1, \operatorname{Ad} 2, \operatorname{Ad} 3$, Ad18, Ad22, Aw1, Aw2, Aw6, Aw8, Aw15, Aw16, Aw19\}; Community 2 (density = .7500): \{Ad8, Ad15, Ad16, Aw9, Aw18, G3, G5, G13, G14\}; Community 3 (density = .8571): $\{$ Ad15, Ad16, Ad19, G1, G3, G12, G14\}; Community 4 (density = .8571): $\{$ Ad14, G2, G6, G7, G8, 
G10, G15 $\}$; Community 5 (density = .8571): $\{$ Ad3, Ad4, Aw3, Aw4, Aw5, Aw6, Aw19\};

Community 6 (density = .8611): $\{$ Ad5, Ad11, Ad16, Ad17, Ad19, Ad20, Ad21, Aw14, G9 $\}$;

Community $7($ density $=.8667):\{\operatorname{Ad} 1, \operatorname{Ad} 5, \operatorname{Ad} 6, \operatorname{Ad} 7, \operatorname{Aw} 7$, Aw9 $\} ;$ Community $8($ density $=$ 1.00000): $\{$ Ad9, Ad12, Ad19, Aw11, Aw12, Aw13\}; Community 9 (density = 1.0000): $\{\operatorname{Ad} 12$, Ad14, Aw17, G4, G11 $\}$; Community 10 (density = 1.0000): $\{$ Ad9, Ad10, Ad11, Ad13, Aw18 $\}$; and Community $11($ density $=1.0000)$ : $\{$ Ad3, Aw3, Aw8, Aw10, Aw15 $\}$.

\section{Conclusions}

\subsection{Summary}

The walktrap (Pons \& Latapy, 2005) and spin-glass (Reichardt \& Bornholdt, 2006) algorithms have been the most popular methods for community detection in network psychometrics. These methods produce partitions of the vertices of the network graph that allow no overlap of the communities. This limitation has been documented in the literature and clique percolation (Farkas et al., 2007; Palla et al., 2005) has been proffered as a useful approach for discovering overlapping communities (Blanken et al., 2018). Nevertheless, Fortunato (2010) has documented several limitations of clique percolation that include: (1) dense communities are not necessarily obtained, (2) many vertices are often not assigned to communities, and (3) the selection of clique size and intensity threshold is somewhat ad hoc. Moreover, it is important to recognize that clique percolation was originally developed for networks that are much larger and sparser than those commonly encountered in network psychometrics.

The limitations of clique percolation notwithstanding, we believe that cliques are an important tool for discovering overlapping community structure in psychological networks. With this principle in mind, we have proposed two alternative heuristic procedures, $\mathrm{MCHOC}$ and GCHOC, which incorporate maximal clique and graph coloring methods, respectively. Because 
these heuristics emphasize an initial focus on the largest cliques, they are more likely to preserve the dense community structure described by Fortunato (2010). MCHOC and GCHOC also drop the intensity threshold requirement associated with clique percolation because intensity is not the only relevant measure of the importance of a clique and, sometimes, cliques of smaller size and intensity have greater clique degree centrality than larger, more intense cliques.

We demonstrated MCHOC and GCHOC using four examples from the network psychometrics literature. Although MCHOC and GCHOC provide similar communities, the latter method tends to yield a finer structure with more communities of greater density because the set of cliques is restricted to those that work well in conjunction with other cliques when partitioning the vertices.

\subsection{Limitations and Extensions}

Unlike clique percolation, parameter selection is not a formidable challenge when using MCHOC and GCHOC. However, the communities identified by the algorithms can be affected by the order in which the cliques are evaluated. We recommend running the algorithms multiple times using different orderings of the cliques and examining the relative densities of the communities to select a solution for interpretation. The exploration of a few different community structures has practical merit; however, the development of specific rules for choosing a solution from among the alternative structures would also have value.

MCHOC and GCHOC might not be particularly useful for very sparse psychological network graphs. If the graph is sparse, then the maximum cliques are potentially small and less interesting. Moreover, a large number of colors might be necessary to assure that no two vertices that do not share an edge in the graph have the same color. The methods are also potentially of limited value if the network is too dense. As the size of the maximum clique approaches $n$, it 
becomes more difficult to identify meaningful distinct communities. Nevertheless, it would seem that MCHOC and GCHOC would be useful for a wide range of psychological networks.

$$
\text { Appendix - Algorithm for Generating All p-colorings }
$$

The required inputs for the graph-coloring algorithm used in the MATLAB implementation of GCHOC (gchoc.m) are: (1) the network graph, $G(V, E)$, for the $n$ vertices/items, and (2) the desired number of colors $(p)$. The underlying optimization problem is to find all partitions, $\Gamma$, of the vertex set $V$ into $p$ subsets, such that a partition, $\gamma=\left\{V_{1}, \ldots, V_{p}\right\}$, is a member of $\Gamma$ if the vertices of every subset form a clique.

The algorithm used to generate all $p$-colorings makes use of an implicit enumeration procedure. This is much faster than explicit enumeration of all possible assignments of $p$ colors to $n$ items. The number of ways to assign $p$ colors to $n$ items is a Stirling number of the second kind and grows rapidly as a function of $n$ and $p$. For example, for the Obama network, the number of ways to assign $p=2$ colors to $n=10$ items is only 511 ; however, for the positive emotions network, the number of ways to assign $p=12$ colors to $n=56$ items is approximately $5.16 \times 10^{51}$. In the latter example, explicit enumeration of such a large number of colorings is infeasible. Implicit enumeration reduces the computational burden by avoiding the explicit generation of many colorings. For example, if items 1 and 2 do not share an edge, then all possible colorings where items 1 and 2 share the same color can be implicitly eliminated from consideration and their explicit generation is unnecessary.

The implicit enumeration algorithm is similar to one originally proposed by Brown (1972). Algorithmic descriptions for closely related problems are also available from several sources in the literature (Brusco, 2003; Brusco \& Cradit, 2004; Brusco \& Stahl, 2005; Hansen \& Delattre, 1978; Klein \& Aronson, 1991). The algorithm uses a depth-first search strategy. Vertices are 
considered in turn for color assignment and the position pointer, $j$, marks the vertex currently under consideration for assignment. A complete coloring occurs when all vertices have been assigned a color, whereas a partial coloring corresponds to $j<n$ color assignments. The computational efficiency of the algorithm improves when partial colorings can be eliminated (or pruned) when $j$ is small, which avoids the need to pursue coloring assignments that cannot possibly lead to an optimal solution. The pruning implicitly circumvents the need to generate a large number of complete colorings that cannot possibly be feasible. It is computationally advantageous to place weakly-connected vertices (i.e., those associated with fewer edges) early in the vertex list because such vertices are more constrained with respect to their color assignments (i.e., there many vertices that cannot have the same color as the weakly-connected vertices). Therefore, we re-sequence the vertices in ascending order based on their degree centrality.

Other notation that will be used in the algorithm description includes: (i) $\lambda$, the number of colors that have not yet been used and (ii) $m$, the color being considered for assignment to the current vertex. The specific steps of the algorithm are as follows:

Step 0. Initialization. Reorder the vertices is ascending order of their degree centrality. Set $j=0, \lambda=K, \Gamma=\varnothing$, and $\gamma=\left\{V_{1}, \ldots, V_{p}\right\}=\varnothing$. Go to Step 1 .

Reordering the symptoms based on degree centrality facilitates faster pruning. Parameter values are also initialized.

Step 1. Branch forward. Set $m=1$ and $j=j+1$. Go to Step 2 .

The branch forward step increments the pointer $(j)$ to the next vertex and resets the color index (m) to the first color. 
Step 2. Color assignment. Set $V_{m}=V_{m} \cup\{j\}$. If $\left|V_{m}\right|=1$, then set $\lambda=\lambda-1$. Go to Step 3.

The current vertex is assigned to the subset associated with the current color. If it is the first vertex assigned to that color (i.e., $\left|V_{m}\right|=1$ ), then the number of unused colors is reduced by one via $\lambda=\lambda-1$.

Step 3. Remaining colors test. If $\lambda>n-j$, go to Step 8; otherwise, go to Step 4 If the number of unused colors $(\lambda)$ exceeds the number of vertices remaining to be assigned $(n-$ $j)$, then the current partial coloring must be pruned because there are not enough remaining vertices to accommodate the empty colors. In this situation, control is passed to Step 8 for dispensation of the partial coloring.

Step 4. Assigned vertex threshold test: If $\{h, j\} \notin E$, for any $h<j: h \in V_{m}$, then go to Step 8; otherwise, go to Step 5.

If the current vertex $j$ shares a color with any of the previously assigned vertices $(1 \leq h \leq j-1)$ and there is no edge connecting $h$ and $j$ (i.e., $\{h, j\} \notin E\}$, then the partial coloring should be pruned (control passed to Step 8) because it cannot possibly lead to a feasible $p$-coloring.

Step 5. Unassigned vertex test: Set $\xi_{i q}=1$ if $\{h, i\} \notin E$ for any $h \in V_{q}$ and 0 otherwise, for all $j+1 \leq i \leq n, 1 \leq q \leq p$. Set $\xi_{i}=\prod_{q-1}^{p} \xi_{i q}$, for all $j+1 \leq i \leq n$. If $\xi_{i}=1$ for any $j+1 \leq i$ $\leq n$, then go to Step 8; otherwise, go to Step 6 .

For vertices that have not yet been assigned $(j+1 \leq i \leq n)$ the unassigned vertex test requires the assessment of a feasible coloring. The variable $\xi_{i q}$ assumes a value of one if the assignment of color $q$ to vertex $i$ will result in a missing edge between $i$ and one or more other vertices $h \in V_{q}$. The variable $\xi_{i}$ is the product of $\xi_{i q}$ across all $q$. If $\xi_{i}=1$, then there is no possible color 
assignment for $\mathrm{i}$ that will not result in a missing edge and, therefore, the partial solution should be pruned.

Step 6. Complete coloring? If $p<n$, then go to Step 2; otherwise, go to Step 7. If $p<n$, then the coloring is only partial and control returns to Step 2 for assignment of the next vertex. If $p=n$, then the coloring is complete and control passes to Step 7.

Step 7. Append to the set of p-colorings. Set $\Gamma=\Gamma \cup \gamma$. Go to Step 8.

Step 7 is reached only for complete colorings and assures that the current coloring, $\gamma$, is appended to the list of possible colorings, $\Gamma$.

Step 8. Dispensation. If $(m=p) \vee\left|V_{m}\right|=1$, then go to Step 10; otherwise, go to Step 9 . A determination is made at Step 8 as to whether the control should proceed to branch right (Step 9) or depth retraction (Step 10) Branching right, which means assigning the current vertex $j$ to the next available color, cannot occur if either (i) $m=p$, which means all colors have been tested for the vertex, or (ii) removing the color assignment will make that color unused.

Step 9. Branch right (vertex recolor). Set $V_{m}=V_{m}-\{j\}$. If $\left|V_{m}\right|=0$, then set $\lambda=\lambda+1$. Set $m=m+1, V_{m}=V_{m} \cup\{j\}$. If $\left|V_{m}\right|=1$, then set $\lambda=\lambda-1$. Return to Step 3 . Branching right requires the removal of the current coloring $(m)$ of vertex $j$, and replacement with the new coloring $(m+1)$. Any required updating of the number of unused colors $(\lambda)$ resulting from these modifications is also implemented.

Step 10. Depth retraction. Set $V_{m}=V_{m}-\{j\}$. If $\left|V_{m}\right|=0$, then set $\lambda=\lambda+1$. Set $j=j-1$. If $j>0$, then go to Step 8; otherwise return the set of $p$-colorings of the network graph, $\Gamma$, and Stop.

Like branching right, depth retraction also begins with the removal of the current coloring $(m)$ of vertex $j$. However, in the case of depth retraction, this is followed by retracting or "backing-up" 
to the previous vertex via $j=j-1$. When retraction results in $j=0$, all colorings have been either implicitly or explicitly evaluated and the algorithm terminates with the set of all $p$-colorings of the network graph. If $\Gamma$ is empty upon termination, then a $p$-coloring of the network is infeasible and the algorithm should be re-executed after increasing the value of $p$ by one.

With some minor modifications, the algorithm for generating all $p$-colorings can be adapted for the much simpler problem of finding all maximal cliques of size $k_{\min }$ or larger. This adapted version of the algorithm is used in the MATLAB implementation of MCHOC (mchoc . m). The set of all maximal cliques, $\Phi$, should be initialized to $\Phi=\varnothing$ in Step 0 . The value of $p$ is set to two, where subset one will contain vertices in the clique and subset two will contain vertices not in the clique. Step 4 of the algorithm is modified so that the test is only performed for subset $m=$ 1 because the clique condition does not have to be satisfied for subset $m=2$. Step 5 of the algorithm is eliminated. Step 7 is modified so that a clique $\phi$ is only appended to $\Phi$ if it contains at least $k_{\min }$ vertices and is maximal. Accordingly, the conditions necessary for adding $\phi$ to $\Phi$ are: (i) $|\phi| \geq k_{\min }$, and (ii) $\phi \not \subset \phi^{\prime}$ for all $\phi^{\prime} \in \Phi$. Finally, the $\left|V_{m}\right|=1$ condition is removed from the dispensation test in Step 8 because, in this adapted version of the algorithm, the subset labels are not interchangeable (i.e., subset one explicitly refers to vertices in the clique, whereas subset two explicitly refers to vertices not in the clique). 


\section{References}

Adamcsek, B., Palla, G., Farkas, I. J., Derényi, I., \& Vicsek, T. (2006). CFinder: locating cliques and overlapping modules in biological networks. Bioinformatics, 22 (8), 1021-1023.

Appel, K., \& Haken, W. (1977a). Every planar map is four colorable. I. Discharging. Illinois Journal of Mathematics, 21(3), 429-490.

Appel, K., \& Haken, W. (1977b). Solution of the four color map problem. Scientific American, 237(4), 108-121.

Ashkin, J., \& Teller, E. (1943). Statistics of two-dimensional lattices with four components. Physical Review, 64(5-6), 178-184.

Baker, F. B., \& Hubert, L. J. (1976). A graph-theoretic approach to goodness of fit in completelink hierarchical clustering. Journal of the American Statistical Association, 71(356), 870-878.

Blanken, T. F., Deserno, M. K., Dalege, J., Borsboom, D., Blanken, P., Kerkhof, G. A., \& Cramer, A. O. J. (2018). The role of stabilizing and communicating symptoms given overlapping communities in psychopathology networks. Scientific Reports, 8, 59. doi:10.1038/s41598-018-24224-2

Bomze, I. M., Budinich, M., Pardalos, P. M., \& Pelillo, M. (1999). The maximum clique problem. In D.-Z. Du \& P. M. Pardalos (Eds.), Handbook of Combinatorial Optimization, Vol. 4, (pp. 1-74), Boston: Kluwer.

Borsboom, D., Fried, E. I., Epskamp, S., Waldrop, L. J., van Borkulo, C. D., van der Maas, H. L. J. (2017). False alarm? A comprehensive reanalysis of "evidence that psychopathology symptom networks have limited replicability" by Forbes, Wright, Markon, and Krueger, (2017). Journal of Abnormal Psychology, 126 (7), 989-999.

Briganti, G., Kempenaers, C., Braun, S., Fried, E. I., Linkowski, P. (2018). Network analysis of empathy items from the interpersonal reactivity index in 1973 young adults. Psychiatry Research, 265, 87-92.

Broadbent, S., \& Hammersley, J. (1957). Percolation processes: I. Crystals and mazes. Proceedings of the Cambridge Philosophical Society, 53(3), 629-641.

Brooks, R. L. (1941). On colouring the nodes of a network. Proceedings of the Cambridge Philosophical Society, 37(2), 194-197.

Brown, J. R. (1972). Chromatic scheduling and the chromatic number problem. Management Science, 19(4, part 1), 456-463.

Brusco, M. J. (2003). An enhanced branch-and-bound algorithm for a partitioning problem. British Journal of Mathematical and Statistical Psychology, 56(1), 83-92.

Brusco, M. J., \& Cradit, J. D. (2004). Graph coloring, minimum-diameter partitioning, and the analysis of confusion matrices. Journal of Mathematical Psychology, 48(5), 301-309.

Brusco, M. J., Davis-Stober, C. P., \& Steinley, D. (2021). Ising formulations of some graphtheoretic problems in psychology: models and methods. Journal of Mathematical Psychology, 102 (June), Article 102536. https://doi.org/10.1016/j.jmp.2021.102536 
Brusco, M. J., \& Stahl, S. (2005). Branch-and-bound applications in combinatorial data analysis. New York: Springer.

Brusco, M. J., \& Steinley, D. (2014). Model selection for minimum-diameter partitioning. British Journal of Mathematical and Statistical Psychology, 67(3), 471-495.

Brusco, M. J., Steinley, D., Hoffman, M., Davis-Stober, C., \& Wasserman, S. (2019). On Ising models and algorithms for the construction of symptom networks in psychopathology research. Psychological Methods, 24 (6), 735-753.

Choi, K. W., Batchelder, A. W., Ehlinger, P. P., Safren, S. A., \& O’Cleirigh, C. (2017). Applying network analysis to psychological comorbidity and health behavior: Depression, PTSD, and sexual risk in sexual minority men with trauma histories. Journal of Consulting and Clinical Psychology, 85, 1158-1170.

Christensen, A. P. (2018). NetworkToolbox: Methods and measures for brain, cognitive, and psychometric network analysis in R. The R Journal, 10 (2), 422-439. Retrieved 2/17/2021 from: https://journal.r-project.org/archive/2018/RJ-2018-065/RJ-2018-065.pdf

Cosgrove, A. L., Kenett, Y. N., Beaty, R. E., \& Diaz, M. T. (2021). Quantifying flexibility in thought: The resiliency of semantic networks differs across the lifespan. Cognition, 211, Article 104631, 1-11. https://doi.org/10.1016/j.cognition.2021.104631

Dailey, D. P. (1978). Graph coloring by humans and machines: A polynomial complete problem solving task. Doctoral dissertation, University of Colorado.

Dalege, J., Borsboom, D., van Harreveld, F., \& van der Maas, H. J. L. (2017). Network analysis on attitudes: a brief tutorial. Social Psychological and Personality Science, 8 (5), 528537.

Epskamp, S., Cramer, A. O., Waldorp, L. J., Schmittmann, V. D., \& Borsboom, D. (2012). qgraph: Network visualizations of relationships in psychometric data. Journal of Statistical Software, 48, 1-18.

Epskamp, S., \& Fried, E. I. (2018). A tutorial on regularized partial correlation networks. Psychological Methods, 23(4), 617-634.

Farkas, I. J., Ábel, D., Palla, G., \& Vicsek, T. (2007). Weighted network modules. New Journal of Physics, 9(180), 1-18.

Forbes, M. K., Wright, A. G. C., Markon, K. E., \& Krueger, R. F. (2017). Evidence that psychopathology symptom networks have limited replicability. Journal of Abnormal Psychology, 126 (7), 969-988.

Fortuin, C. M., \& Kasteleyn, P. W. (1972). On the random-cluster model: I. Introduction and relation to other models. Physica, 57, 536-564.

Fortunato, S. (2010). Community detection in graphs. Physics Reports, 486, 75-174.

Fried, E. I. (2016). R tutorial: how to identify communities of items in networks. Retrieved from http://psych-networks.com/r-tutorial-identify-communities-items-networks/

Friedman, J. H., Hastie, T., \& Tibshirani, R. (2008). Sparse inverse covariance estimation with the graphical lasso. Biostatistics, 9(3), 432-441. 
Friedman, J. H., Hastie, T., \& Tibshirani, R. (2014). glasso: Graphical lasso-estimation of Gaussian graphical models (R package version 1.8). Retrieved from https://CRAN.Rproject.org/package_glasso

Garey, M. R., \& Johnson, D. S. (1979). Computers and intractability: A guide to the theory of NP-completeness. New York: W. H. Freeman.

Gates, K. M., Henry, T., Steinley, D., \& Fair, D. A. (2016). A Monte Carlo evaluation of weighted community detection algorithms. Frontiers in Neuroinformatics, 10, 45. doi: 10.3389/fninf.2016.00045

Golino, H., \& Demetriou, A. (2017). Estimating the dimensionality of intelligence like data using exploratory graph analysis. Intelligence, 62, 54-70.

Golino, H. F., \& Epskamp, S. (2017). Exploratory graph analysis: A new approach for estimating the number of dimensions in psychological research. PloS One, 12(6), :e0174035, 2017. URL https://doi.org/10.1371/journal.pone.0174035.

Guthrie, F. (1880). Note on the colouring of maps. Proceedings of the Royal Society of Edinburgh, 10, 727-728.

Hansen, P., \& Delattre, M. (1978). Complete-link cluster analysis by graph coloring. Journal of the American Statistical Association, 73(362), 397-403.

Harary, F., \& Ross, I. C. (1957). A procedure for clique detection using the group matrix. Sociometry 20, 205-215.

Hevey, D. (2018). Network analysis: a brief overview and tutorial. Health Psychology and Behavioral Medicine, 6(1), 301-328.

Hoffman, M., Steinley, D., Gates, K. M., Prinstein, M. J., \& Brusco, M. J. (2018). Detecting clusters/communities in social networks. Multivariate Behavioral Research, 53(1), 57-73.

Hubert, L. J. (1974a). Some applications of graph theory to clustering. Psychometrika, 39(3), 283-309.

Hubert, L. (1974b). Spanning trees and aspects of clustering. British Journal of Mathematical and Statistical Psychology, 27(1), 14-28.

Ising, E. (1925). Beitrag zur theorie des ferromagnetismus. Zeitschrift für Physik, 31(1), 253258.

Jones, P. J., Ma, R., \& McNally, R. J. (2019). Bridge centrality: A network approach to understanding comorbidity. Multivariate Behavioral Research. doi:10.1080/00273171.2019.1614898

Jones, P. J., Mair, P., Riemann, B. C., Mungo, B. L., \& McNally, R. J. (2018). A network perspective on comorbid depression in adolescents with obsessive-compulsive disorder. Journal of Anxiety Disorders, 53, 1-8. http://dx.doi.org/10.1016/j.janxdis.2017.09.008

Kaiser, T., Herzog, P., Voderholzer, U., \& Brakemeier, E. (2021). Unraveling the comorbidity of depression and anxiety in a large inpatient sample: Network analysis to examine bridge symptoms. Depression \& Anxiety, 38, 307-317. 
Kempe, A. B. (1879). On the geographical problem of the four colours. American Journal of Mathematics, the Johns Hopkins University Press, 2(3), 193-220.

Kendler, K. S., Aggen, S. H., Flint, J., Borsboom, D., \& Fried, E. I. (2018). The Centrality of DSM and non-DSM Depressive Symptoms in Han Chinese Women with Major Depression. Journal of Affective Disorders, 227, 739-744.

Kessler, R. C., Berglund, P., Chiu, W. T., Demler, O., Heeringa, S., Hiripi, E.,. . . Zheng, H. (2004). The US National Comorbidity Survey Replication (NCS-R): Design and field procedures. International Journal of Methods in Psychiatric Research, 13, 69-92.

Klein, G., \& Aronson, J. E. (1991). Optimal clustering: A model and method. Naval Research Logistics, 38(3), 447-461.

Kruis, J., \& Maris, G. (2016). Three representations of the Ising model. Scientific Reports, 6(34175), 1-11. doi: 10.1038/srep34175

Lange, J. (2019). Package 'CliquePercolation'. Retrieved from: https://cran.rproject.org/web/packages/CliquePercolation/CliquePercolation.pdf

Lange, J., \& Zickfeld, J. H. (2021). Emotions as overlapping causal networks of emotion components: Implications and methodological approaches. Emotion Review, 13(2), 157167.

Lucas, A. (2014). Ising formulations of many NP problems. Frontiers in Physics, 2, article 5, Retrieved from: https://www.frontiersin.org/articles/10.3389/fphy.2014.00005/full

Luce, R. D. (1950). Connectivity and generalized cliques in sociometric group structure. Psychometrika, 15(2), 169-190.

Marsman, M., Borsboom, D., Kruis, J., Epskamp, S., van Bork, R., Waldorp, L. J., van der Maas, H. L. J., \& Maris, G. (2018). An introduction to network psychometrics: Relating Ising models to item response theory models. Multivariate Behavioral Research, 53(1), 15-35.

Marsman, M., Maris,G., Bechger, T., \& Glas, C. (2015). Bayesian inference for low-rank Ising networks. Scientific Reports, 5(9050), 1-7. doi: 10.1038/srep09050

MATLAB. (2020). version 9.8.0 (R2020a). Natick, Massachusetts: The MathWorks Inc.

May, K. O. (1965). The origin of the four-color conjecture. Isis, 56(3), 346-348. Retrieved February 27, 2020, from www.jstor.org/stable/228109

McElroy, E., \& Patalay, P. (2019). In search of disorders: internalizing symptom networks in a large clinical sample. Journal of Child Psychology and Psychiatry, 60 (8), 897-906.

Méndez-Díaz, I., \& Zabala, P. (2006). A branch-and-cut algorithm for graph coloring. Discrete Applied Mathematics, 154, 826-847.

Méndez-Díaz, I., \& Zabala, P. (2008). A cutting plane algorithm for graph coloring. Discrete Applied Mathematics, 156, 159-179.

Palla, G., Derényi, I., Farkas, I. \& Vicsek, T. (2005). Uncovering the overlapping community structure of complex networks in nature and society. Nature, 435, 814-818.

Pardalos, P. M., \& Xue, J. (1994). The maximum clique problem. Journal of Global Optimization, 4(3), 301-328. 
Peay, E. R. (1975). Nonmetric grouping: clusters and cliques. Psychometrika, 40 (3), 297-313.

Pons, P., \& Latapy, M. (2005). Computing communities in large networks using random walks (Long Version). Cornell University Library. Physics and Society.

Potts, R. (1952). Some generalized order-disorder transformations. Mathematical Proceedings of the Cambridge Philosophical Society, 48(1), 106-109.

Reichardt, J., \& Bornholdt, S. (2006). Statistical mechanics of community detection. Physical Review E: Statistics and Nonlinear Soft Matter Physics, 74, 016110-1-016110-14

Savi, A. O., Marsman, M., van der Maas, H. L. J., \& Maris, G. K. J. (2019). The wiring of intelligence. Perspectives on Psychological Science, 14(6), 1034-1061.

Steinley, D., Hoffman, M., Brusco, M. J., \& Sher, K. J. (2017). A method for making inferences in network analysis: comment on Forbes, Wright, Markon, and Krueger (2017). Journal of Abnormal Psychology, 126 (7), 1000-1010.

Tait, P. G. (1880a). On the colourings of maps. Proceedings of the Royal Society of Edinburgh, 10(4), 501-503.

Tait, P. G. (1880b). Remarks on the previous communication. Proceedings of the Royal Society of Edinburgh, 10, 729.

Tarjan, R. E., \& Trojanowski, A. E. (1977). Finding a maximum independent set. SIAM Journal on Computing, 6, 537-546. https://doi.org/10.1137/0206038

van Borkulo, C. D., Borsboom, D., Epskamp, S., Blanken, T. F., Boschloo, L., Schoevers, R. A., \& Waldorp, L. J. (2014). A new method for constructing networks from binary data. Scientific Reports, 4(5918), 1-10. doi:10.1038/srep05918.

van der Kloot, W. A., Spaans, A. M. J., \& Heiser, W. J. (2005). Instability of hierarchical cluster analysis due to input order of the data: The PermuCLUSTER solution. Psychological Methods, 10(4), 468-476.

Vogiatzis, C., Veremyev, A., Pasiliao. E. L., \& Pardalos, P .M. (2015). An integer programming approach for finding the most and the least central cliques. Optimization Letters, 9, 615633.

von Luxburg, U. (2007). A tutorial on spectral clustering. Statistics and Computing, 17 (4), 395416.

Weidman, A. C., \& Tracy, J. L. (2020). Picking up good vibrations: Uncovering the content of distinct positive emotion subjective experience. Emotion, 20(8), 1311-1331.

Williams, D. R., \& Rast, P. (2020). Back to the basics: Rethinking partial correlation network methodology. British Journal of Mathematical and Statistical Psychology, 73(2), 187212.

Williams, D. R., Rhemtulla, M., Wysocki, A. C., \& Rast, P. (2019). On nonregularized estimation of psychological networks. Multivariate Behavioral Research, 54 (5), 719 750. 
Table 1. Eight 2-colorings of the Obama network graph and the average intensity of the cliques.

\begin{tabular}{lccc}
\hline & Clique 1 & Clique 2 & Average Intensity \\
\hline Coloring 1 & $\{1,2,3,4,5,6,10\}$ & $\{7,8,9\}$ & .7757 \\
Coloring 2 & $\{2,3,6,7,8,9\}$ & $\{1,4,5,10\}$ & .6407 \\
Coloring 3 & $\{1,3,4,5,6,10\}$ & $\{2,7,8,9\}$ & .7001 \\
Coloring 4 & $\{1,2,4,5,6,10\}$ & $\{3,7,8,9\}$ & .6942 \\
Coloring 5 & $\{1,2,3,4,5,10\}$ & $\{6,7,8,9\}$ & .6552 \\
Coloring 6 & $\{1,4,5,6,10\}$ & $\{2,3,7,8,9\}$ & .6665 \\
Coloring 7 & $\{1,2,4,5,10\}$ & $\{3,6,7,8,9\}$ & .6540 \\
Coloring 8 & $\{1,3,4,5,10\}$ & $\{2,6,7,8,9\}$ & .6525 \\
\hline
\end{tabular}


Table 2. Maximal cliques $(k \geq 3)$ for the NCS-R network.

\begin{tabular}{|c|c|c|c|}
\hline & Clique & & Clique \\
\hline Clique memberships & Size & Intensity & Centrality \\
\hline$C_{7}^{1}$ - 1:Depressed mood, 2:Loss of interest, 3:Weight problems, 4:Sleep problems, 5:Psychomotor disturbances, 7:Self-reproach, 8:Concentration problems & 7 & .8868 & 9 \\
\hline$C_{7}^{2}$ - 1:Depressed mood, 2:Loss of interest, 3:Weight problems, 4:Sleep problems, 7:Self-reproach, 8:Concentration problems, 9:Suicidal ideation & 7 & .8447 & 8 \\
\hline$C_{7}^{3}$ - 1:Depressed mood, 2:Loss of interest, 3:Weight problems, 4:Sleep problems, 6:Fatigue, 7:Self-reproach, 8:Concentration problems & 7 & .9154 & 8 \\
\hline$C_{7}^{4}-12$ :No control over anxiety, 13:Feeling on edge, 14:Fatigue, 15:Irritability, 16:Concentration problems, 17:Muscle tension, 18:Sleep problems & 7 & .8397 & 8 \\
\hline$C_{7}^{5}-11$ :Anxiety about $>1$ event. 12:No control over anxiety, 13:Feeling on edge, 14:Fatigue, 15:Irritability, 16:Concentration problems, 18:Sleep problems & 7 & 1.2303 & 9 \\
\hline$C_{5}^{6}$ - 5:Psychomotor disturbances, 14:Fatigue, 15:Irritability, 16:Concentration problems, 17:Muscle tension & 5 & .4330 & 12 \\
\hline$C_{5}^{7}$-5:Psychomotor disturbances, 7:Self-reproach, 14:Fatigue, 16:Concentration problems, 17:Muscle tension & 5 & .2988 & 13 \\
\hline$C_{5}^{8}-7:$ Self-reproach, 12: No control over anxiety, 14:Fatigue, 16:Concentration problems, 17:Muscle tension & 5 & .3533 & 13 \\
\hline$C_{4}^{9}$ - 6:Fatigue, 7:Self-reproach, 8:Concentration problems, 16:Concentration problems & 4 & .3746 & 14 \\
\hline$C_{4}^{10}$ - 1:Depressed mood, 5:Psychomotor disturbances, 7:Self-reproach, 10:Chronic anxiety/worry & 4 & .5024 & 12 \\
\hline$C_{4}^{11}$ - 1:Depressed mood, 7:Self-reproach, 9:Suicidal ideation, 10:Chronic anxiety/worry & 4 & .6971 & 11 \\
\hline$C_{4}^{12}-5$ :Psychomotor disturbances, 7:Self-reproach, 8:Concentration problems, 16:Concentration problems & 4 & .3639 & 14 \\
\hline$C_{4}^{13}$ - 6:Fatigue, 14:Fatigue, 16:Concentration problems, 18:Sleep problems & 4 & .5720 & 12 \\
\hline$C_{4}^{14}-6:$ Fatigue, $7:$ Self-reproach, 14:Fatigue, 16:Concentration problems & 4 & .3497 & 14 \\
\hline$C_{4}^{15}-1$ :Depressed mood, 2:Loss of interest, 7:Self-reproach, 12:No control over anxiety & 4 & .4535 & 14 \\
\hline$C_{4}^{16}$ - 4:Sleep problems, 6:Fatigue, 18:Sleep problems & 3 & .5519 & 14 \\
\hline
\end{tabular}

Note. Cliques $C_{7}^{1}, C_{7}^{2}$, and $C_{7}^{3}$ are 6-adjacent and form Community 1 . Cliques $C_{7}^{4}$ and $C_{7}^{5}$ are 6-adjacent and form Community 2. Together, these five 7-cliques placed 17 of the 18 symptoms into communities. Symptom 10 joints Community 1 via clique $C_{4}^{10}$, which shares three of its four members with clique $C_{7}^{1}$. 
Table 3. Maximal cliques $(k \geq 3)$ for the Han depression network.

\begin{tabular}{|c|c|c|c|}
\hline Clique memberships & $\begin{array}{c}\text { Clique } \\
\text { size }\end{array}$ & Intensity & $\begin{array}{l}\text { Clique } \\
\text { Centrality }\end{array}$ \\
\hline$C_{5}^{1}$ - 6:worthlessness, 9: $\downarrow$ self-esteem, 10: $\downarrow$ confidence, 16:hopelessness, 18:helplessness & 5 & .6664 & 9 \\
\hline$C_{5}^{2}-4: \downarrow$ motor movement, 6 :worthlessness, $9: \downarrow$ self-esteem, 10: $\downarrow$ confidence, 18 :helplessness & 5 & .4453 & 12 \\
\hline$C_{5}^{3}-4: \downarrow$ motor movement, 9: $\downarrow$ self-esteem, 10: $\downarrow$ confidence, 13: $\downarrow$ libido, 18:helplessness & 5 & .3124 & 12 \\
\hline$C_{5}^{4}-4: \downarrow$ motor movement, 9: $\downarrow$ self-esteem, 13: $\downarrow$ libido, 18:helplessness, 19:nervousness & 5 & .2269 & 13 \\
\hline$C_{5}^{5}-2: \Delta$ weight, $3: \Delta$ sleep, $4: \downarrow$ motor movement, appetite, 5: fatigue, $13: \downarrow$ libido & 5 & .3973 & 11 \\
\hline$C_{5}^{6}-2: \Delta$ weight, appetite, $4: \downarrow$ motor movement, 5: fatigue, $13: \downarrow$ libido, 14:unreactive mood & 5 & .3541 & 13 \\
\hline$C_{5}^{7}-4: \downarrow$ motor movement, 5: fatigue, 7 :difficulty concentrating, $13: \downarrow$ libido, 14:unreactive mood & 5 & .4122 & 12 \\
\hline$C_{5}^{8}-4: \downarrow$ motor movement, 7:difficulty concentrating, 10: $\downarrow$ confidence, $13: \downarrow$ libido, 14:unreactive mood & 5 & .5042 & 12 \\
\hline$C_{5}^{9}-4: \downarrow$ motor movement, 6:worthlessness, 7:difficulty concentrating, 10: $\downarrow$ confidence, 14 :unreactive mood & 5 & .4709 & 13 \\
\hline$C_{5}^{10}-4: \downarrow$ motor movement, 6 :worthlessness, 8:suicidality, 10: $\downarrow$ confidence, 14:unreactive mood & 5 & .3287 & 13 \\
\hline$C_{5}^{11}$ - 6:worthlessness, 8:suicidality, 10: $\downarrow$ confidence, 14:unreactive mood, 16:hopelessness & 5 & .4584 & 12 \\
\hline$C_{5}^{12}-4: \downarrow$ motor movement, 5: fatigue, 7:difficulty concentrating, 13: $\downarrow$ libido, 19:nervousness & 5 & .3337 & 12 \\
\hline$C_{4}^{13}$ - 6:worthlessness, 8 :suicidality, 16 :hopelessness, 17 :crying & 4 & .3408 & 14 \\
\hline$C_{4}^{14}$ - 6:worthlessness, 16:hopelessness, 17:crying, 18:helplessness & 4 & .2652 & 14 \\
\hline$C_{4}^{15}-4: \downarrow$ motor movement, 9: $\downarrow$ self-esteem, 12:worse in AM, 13: $\downarrow$ libido & 4 & .2186 & 13 \\
\hline$C_{4}^{16}-4: \downarrow$ motor movement, 5 : fatigue, 12 :worse in AM, $13: \downarrow$ libido & 4 & .4178 & 12 \\
\hline$C_{4}^{17}-2: \Delta$ weight, appetite, $3: \Delta$ sleep, $4: \downarrow$ motor movement, 8:suicidality & 4 & .3073 & 13 \\
\hline$C_{4}^{18}-2: \Delta$ weight, appetite, $4: \downarrow$ motor movement, 8:suicidality, 14:unreactive mood & 4 & .2837 & 12 \\
\hline$C_{4}^{19}-4: \downarrow$ motor movement, 8:suicidality, 11:distinct quality of depressed mood, 14:unreactive mood & 4 & .3800 & 12 \\
\hline$C_{4}^{20}-9: \downarrow$ self-esteem, 16:hopelessness, 18:helplessness, 19:nervousness & 4 & .3935 & 11 \\
\hline$C_{3}^{21}-1: \downarrow$ interest, 14:unreactive mood, 16:hopelessness & 3 & .4811 & 13 \\
\hline$C_{3}^{22}-2: \Delta$ weight, appetite, 8 :suicidality, 17 :crying & 3 & .2175 & 11 \\
\hline
\end{tabular}


Table 4. A two-community and four-community solution for the Han depression network.

Two-Community Solution

Community 1: $1: \downarrow$ interest, 2: $\Delta$ weight, appetite, $3: \Delta$ sleep, 4: $\downarrow$ motor movement, 5: fatigue, 6:worthlessness, 7:difficulty concentrating,

(.4417) $\quad 8$ :suicidality, $10: \downarrow$ confidence, 11 :distinct quality of depressed mood, 12:worse in AM, 13: $\downarrow$ libido

14:unreactive mood, 16:hopelessness, 17:crying, 19:nervousness

Community 2: 6:worthlessness, 9: $\downarrow$ self-esteem, 10: $\downarrow$ confidence, 16:hopelessness, 18:helplessness (1.0000)

Four-Community Solution

Community 1: 4: $\downarrow$ motor movement, 6:worthlessness, 9: $\downarrow$ self-esteem, 10: $\downarrow$ confidence, 12:worse in AM, 13: $\downarrow$ libido, 16:hopelessness, (.7222) 18:helplessness, 19:nervousness

Community 2: 1: $\downarrow$ interest, 6:worthlessness, 8:suicidality, 10: $\downarrow$ confidence, 14:unreactive mood, 16:hopelessness, 17:crying (.7143)

Community 3: 2: $\Delta$ weight, appetite, 3: $\Delta$ sleep, 4: $\downarrow$ motor movement, 5: fatigue, 7:difficulty concentrating, 10: $\downarrow$ confidence, (.7857) 13: $\downarrow$ libido, 14 :unreactive mood

Community 4: 4: $\downarrow$ motor movement, 8:suicidality, 11:distinct quality of depressed mood, 14:unreactive mood (1.0000)

Note. The density of each community is shown in parentheses. Text coloring is used to highlight symptoms that have membership in multiple communities. 
Table 5. Items for the positive emotions network.

Admiration items:

Ad1 = "I felt as if I had just witnessed a fantastic achievement"

Ad2 = "What I just saw was simply amazing"

Ad3 = "I could not believe what I had just seen"

Ad4 = "My mouth fell open"

Ad5 = "I felt motivated to work harder"

Ad6 = "I felt the need to celebrate someone else's accomplishment"

Ad7 = "I felt energized"

Ad8 = "I felt a sense of warmth"

$\operatorname{Ad} 9=$ "I felt as if I could learn a lot from a specific person"

Ad10 = "I strongly valued a specific person's opinion"

Ad11 = "I felt a desire to become more like a specific person"

Ad12 = "I had a great deal of respect toward a specific person"

Ad13 = "I felt lucky to know a specific person"

Ad14 = "I wanted to say thank you to a specific person"

Ad15 = "I was happy"

Ad16 = "I felt positive about myself"

Ad17 = "I felt a desire to be admired"

Ad18 = "I felt humbled"

Ad19 = "I envied a specific person"

$\operatorname{Ad} 20=$ "I felt small in comparison to a specific person"

Ad21 = "I realized I am inept"

$\operatorname{Ad} 22=$ "I felt admiration"

Awe items

Aw1 = "I was amazed"

Aw2 = "I felt wonder"

Aw3 = "I froze"

Aw4 = "I gasped"

Aw5 = "I was rendered speechless"

Aw6 = "I knew I would remember what I saw for a long time"

Aw7 = "I wanted to take a picture of what I saw"

Aw8 = "I continued to think about what I just saw"

Aw9 = "I saw beauty all around me"

Aw10 = "The situation challenged my understanding of the world"

Aw11 = "I wished to soak in my current experience"

Aw12 = "I was losing track of my day-to-day concerns"

Aw13 = "I wished the moment would last longer"

Aw14 = "I felt slight and insignificant in comparison to my surroundings"

Aw15 = "I felt I was in the presence of something quite out of the ordinary"

Aw16 = "I was inspired by what I saw"

Aw17 = "I felt appreciative toward someone or something"

Aw18 = "I wanted to get close to a specific person or object"

Aw19 = "I felt awe"

Gratitude items

G1 = "I felt fortunate"

G2 = "I felt cared for"

G3 = "I felt comforted"

G4 = "I felt like I had benefited by a specific person's action"

G5 = "I wanted to express thanks"

G6 = "I wanted to help a specific person in return"

G7 = "I felt indebted to a specific person"

G8 = "I didn't want to feel like a burden to a specific person"

G9 = "I felt like a failure relative to a specific person who benefited me"

G10 = "I thought that the specific person who helped me should be acknowledged"

G11 = "I felt appreciative toward a specific person"

G12 = "I was thankful for what just happened to me"

G13 = "I feel that there must be some good in the world"

G14 = "I had a more positive outlook on life"

G15 = "I felt gratitude" 
Table 6. A comparison of the communities for positive emotion network:

\begin{tabular}{|c|c|}
\hline Clique percolation communities: Lange \& Zickfeld (2021) & Communities obtained via MCHOC \\
\hline $\begin{array}{l}\text { Community 1: Ad5, Ad16, Ad17, Ad19, Ad20, Ad21, Aw10, } \\
\begin{array}{ll}(.7091) & \text { Aw14, G7, G8, G9 }\end{array}\end{array}$ & $\begin{array}{l}\text { Community } 1: \text { Ad5, Ad11, Ad16, Ad17, Ad19, Ad20, Ad21, } \\
\begin{array}{ll}(.8611) & \text { Aw14, G9 }\end{array}\end{array}$ \\
\hline 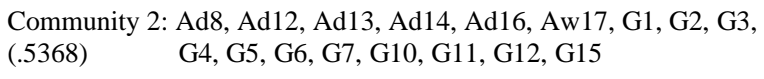 & $\begin{array}{l}\text { Community 2: Ad14, G2, G4, G6, G7, G8, G10, G11 } \\
(.8214)\end{array}$ \\
\hline 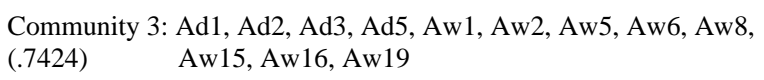 & $\begin{array}{l}\text { Community 3: Ad1, Ad2, Ad3, Ad18, Ad22, Aw1, Aw2, Aw3, Aw4 } \\
\begin{array}{ll}(.5956) & \text { Aw5, Aw6, Aw7, Aw8, Aw10, Aw15, Aw16, Aw19 }\end{array}\end{array}$ \\
\hline $\begin{array}{l}\text { Community 4: G2, G6, G7, G8 } \\
((1.0000)\end{array}$ & $\begin{array}{l}\text { Community 4: Ad14, Aw17, G5, G6, G13 } \\
(1.0000)\end{array}$ \\
\hline $\begin{array}{l}\text { Community 5: Ad3, Ad4, Aw3, Aw4, Aw5 } \\
(1.0000)\end{array}$ & $\begin{array}{l}\text { Community 5: Ad1, Ad4, Ad7, Ad15, Aw7, Aw9 } \\
(1.0000)\end{array}$ \\
\hline $\begin{array}{l}\text { Community 6: Ad2, Ad5, Ad7, Ad15, Ad16, Ad17, Aw2, Aw6, } \\
\begin{array}{ll}(.5934) & \text { Aw7, Aw9, Aw11, Aw12, Aw13, Aw18 }\end{array}\end{array}$ & $\begin{array}{l}\text { Community 6: Ad9, Ad12, Ad19, Aw11, Aw12, Aw13, Aw18, G12 } \\
(.8214)\end{array}$ \\
\hline $\begin{array}{l}\text { Community 7: Ad5, Ad6, Ad9, Ad10, Ad11, Ad12, Ad13, Ad19, } \\
(.5897) \quad \text { Ad20, Ad22, Aw16, Aw18, G14 }\end{array}$ & $\begin{array}{l}\text { Community 7: Ad6, Ad9, Ad10, Ad11, Ad12, Ad13, G2 } \\
(.8571)\end{array}$ \\
\hline $\begin{array}{l}\text { Community 8: Ad8, G3, G13, G14 } \\
(1.0000)\end{array}$ & $\begin{array}{l}\text { Community 8: Ad15, Ad16, Ad19, G1, G3, G14 } \\
(1.0000)\end{array}$ \\
\hline $\begin{array}{l}\text { Community 9: Ad8, Ad15, Ad16, G1, G14 } \\
(.9000)\end{array}$ & $\begin{array}{l}\text { Community 9: Ad8, Ad15, Ad16, Aw9, G3, G5, G13, G14 } \\
\text { (.8214) }\end{array}$ \\
\hline Isolate: Ad18 & $\begin{array}{l}\text { Community 10: Ad8, Ad14, Aw2, Aw5, G15 } \\
(1.0000)\end{array}$ \\
\hline
\end{tabular}

Note. The density of each community is shown in parentheses. 
Input $G(V, E)$ and specify the minimal clique size, $k_{\min }$ (and the number of colors, $p$, if GCHOC used) Generate the candidate cliques as either (i) all maximal cliques of size $k \geq k_{\min }$ for MCHOC, or (ii) using all cliques of size $k \geq k_{\min }$ that are part of a $p$-coloring for GCHOC).

Let the $c$ candidate cliques be denoted $C^{1}, \ldots, C^{c}$.

Initialize the clique community assignment vector, $\chi(d)=0$ for $1 \leq d \leq c$.

Initialize the number of communities $T=0$ and $k_{\max }=\max _{1 \leq d \leq c}\left\{\left|C^{d}\right|\right\}$.

For $k=k_{\max }$ to $k_{\min }$ step -1

flag $1=1$

While flag $1=1$

flag $1=0$

flag $2=1$

While flag $2=1$

flag2 = 0

For all $d^{\prime}=1$ to $c: \chi\left(d^{\prime}\right) \neq 0$

For all $d=1$ to $c:\left|C^{d}\right|=k$

$\bar{V}=V \backslash\left\{\bigcup_{t=1}^{T} H_{t}\right\}$

If $\bar{V} \cap C^{d} \neq \varnothing$ and $\left|C^{d} \cap C^{d}\right|=\left|C^{d}\right|-1$

Set $\chi(d)=\chi\left(d^{\prime}\right)$

Set $H_{\chi(d)}=H_{\chi(d)} \cup C^{d}$

flag $1=1$

flag $2=1$

End if

Next $d$

Next $d^{\prime}$

End while

flag $3=1$

$\mathrm{d}=0$

While flag $3=1$ and $d \leq n$

$d=d+1$

$\bar{V}=V \backslash\left\{\bigcup_{t=1}^{T} H_{t}\right\}$

If $\chi(d)=0$ and $\bar{V} \cap C^{d} \neq \varnothing$

Set $T=T+1$

Set $\chi(d)=E$

Set $H_{T}=H_{T} \cup C^{d}$

flag $3=0$

flag $1=1$

End if

End while

If $V=\left\{\bigcup_{t=1}^{T} H_{t}\right\}$

Return communities $H_{t}(1 \leq t \leq T)$ and STOP.

End if

Next $k$

End While

Return communities $H_{t}(1 \leq t \leq T)$ and STOP.

Figure 1. Pseudocode for MCHOC and GCHOC. 


\begin{tabular}{|c|c|c|c|c|c|c|c|c|c|c|}
\hline & 1 & 4 & 5 & 10 & 2 & 3 & 6 & 8 & 7 & 9 \\
\hline 1 Moral & --- & 0.49 & 1.13 & 0.52 & 0.38 & 1.23 & 1.76 & & & 0.42 \\
\hline 4 Knowedgeable & 0.49 & -- & 2.66 & 0.39 & 1.38 & 0.65 & 0.67 & 0.56 & & \\
\hline 5 Intelligent & 1.13 & 2.66 & -- & 0.25 & 0.58 & 0.68 & 0.86 & 0.31 & & \\
\hline 10 Proud & 0.52 & 0.39 & 0.25 & -- & 0.82 & 0.86 & 0.42 & 2.32 & & \\
\hline 2 Leadership & 0.38 & 1.38 & 0.58 & 0.82 & -- & 0.8 & 0.93 & 0.33 & 0.84 & 0.46 \\
\hline 3 Caring & 1.23 & 0.65 & 0.68 & 0.86 & 0.8 & --- & 1.38 & 0.78 & 0.49 & 0.48 \\
\hline 6 Honest & 1.76 & 0.67 & 0.86 & 0.42 & 0.93 & 1.38 & -- & 0.34 & 0.36 & 0.73 \\
\hline 8 Hopeful & & 0.56 & 0.31 & 2.32 & 0.33 & 0.78 & 0.34 & -- & 0.28 & 0.78 \\
\hline 7 Angry & & & & & 0.84 & 0.49 & 0.36 & 0.28 & -- & 2.21 \\
\hline 9 Afraid & 0.42 & & & & 0.46 & 0.48 & 0.73 & 0.78 & 2.21 & -- \\
\hline
\end{tabular}

Figure 2. Community structure for the Obama network. The borders reveal two maximal 7 cliques and a maximal 6-clique. The two maximal 7-cliques are 6-adjacent and define Community $1\{1,4,5,10,2,3,6,8\}$ as shown via yellow shading. The 6 -clique defines Community $2\{2,3,6,8,7,9\}$ as shown via blue shading. The overlap of the communities is shown via green shading and pertains to a subset of four characteristics $\{2,3,6,8\}$. 


\begin{tabular}{|c|c|c|c|c|c|c|c|c|c|c|c|c|c|c|c|c|c|c|c|}
\hline & & 6 & 9 & 2 & 3 & 4 & 8 & 1 & 5 & 7 & 10 & 11 & 12 & 13 & 14 & 15 & 16 & 18 & 17 \\
\hline Fatigue & 6 & .00 & .00 & 1.18 & .21 & 1.10 & 1.17 & 2.92 & .00 & .49 & .00 & .00 & .00 & .00 & 1.38 & .00 & -.18 & -.20 & .00 \\
\hline Svicidal ideation & 9 & .00 & .00 & .63 & .30 & .45 & .31 & 3.52 & .00 & .94 & .14 & .00 & .00 & .00 & .00 & .00 & .00 & .00 & .00 \\
\hline Loss of interest & 2 & 1.18 & .63 & .00 & .76 & .69 & .97 & 1.93 & .52 & .88 & .00 & .00 & .16 & .00 & .00 & .00 & .00 & .00 & .00 \\
\hline Weight problems & 3 & .21 & .30 & .76 & .00 & 1.39 & .66 & 3.01 & .76 & .15 & .00 & .00 & .00 & .00 & .00 & .00 & .00 & .00 & .00 \\
\hline Sleep problems & 4 & 1.10 & .45 & .69 & 1.39 & .00 & .79 & 3.51 & .66 & .19 & .00 & .00 & .00 & .00 & .00 & .00 & .00 & .77 & .00 \\
\hline Concentration problems & 8 & 1.17 & .31 & .97 & .66 & .79 & .00 & 3.03 & 1.35 & .31 & .00 & .00 & .00 & .00 & .00 & .00 & .97 & .00 & .00 \\
\hline Depressed mood & 1 & 2.92 & 3.52 & 1.93 & 3.01 & 3.51 & $3.03 \Gamma$ & .00 & 1.82 & 1.84 & .53 & .00 & .16 & .00 & .00 & .00 & .00 & .00 & .00 \\
\hline Psychomotor disturbances & 5 & .00 & .00 & .52 & .76 & .66 & 1.35 & 1.82 & .00 & .37 & .10 & .00 & .00 & .00 & -.35 & .12 & .17 & .00 & .16 \\
\hline Self reproach & 7 & .49 & .94 & .88 & .15 & .19 & .311 & 1.84 & .37 & .00 & .261 & .00 & .11 & .00 & .21 & .00 & .09 & .00 & .24 \\
\hline Chronic anxiety/worry & 10 & .00 & .14 & .00 & .00 & .00 & .00 & .53 & .10 & .26 & .001 & 7.03 & .00 & .00 & .00 & .00 & .00 & .00 & .00 \\
\hline Anxiety about $>1$ event & 11 & .00 & .00 & .00 & .00 & .00 & .00 & .00 & .00 & .00 & 7.03 & .00 & 5.90 & 1.96 & 1.67 & 2.43 & 1.89 & 2.20 & .00 \\
\hline No control over anxiety & 12 & .00 & .00 & .16 & .00 & .00 & .00 & .16 & .00 & .11 & .00 & 5.90 & .00 & 1.07 & .66 & .59 & .54 & .59 & .49 \\
\hline Feeling on edge & 13 & .00 & .00 & .00 & .00 & .00 & .00 & .00 & .00 & .00 & .00 & 1.96 & 1.07 & .00 & .74 & 2.02 & 1.60 & 1.61 & .93 \\
\hline Fatigue & 14 & 1.38 & .00 & .00 & .00 & .00 & .00 & .00 & -.35 & .21 & .00 & 1.67 & .66 & .74 & .00 & 1.22 & .82 & .75 & 1.23 \\
\hline Irritability & 15 & .00 & .00 & .00 & .00 & .00 & .00 & .00 & .12 & .00 & .00 & 2.43 & .59 & 2.02 & 1.22 & .00 & 1.41 & .64 & .34 \\
\hline Concentration problems & 16 & -.18 & .00 & .00 & .00 & .00 & 97 & .00 & .17 & .09 & .00 & 1.89 & .54 & 1.60 & .82 & 1.41 & .00 & 1.19 & .36 \\
\hline Sleep problems & 18 & -.20 & .00 & .00 & .00 & .77 & .00 & .00 & .00 & .00 & .00 & 2.20 & .59 & 1.61 & .75 & .64 & 1.19 & .00 & .93 \\
\hline Muscle tension & 17 & .00 & .00 & .00 & .00 & .00 & .00 & .00 & .16 & .24 & .00 & .00 & .49 & .93 & 1.23 & .34 & .36 & .93 & .00 \\
\hline
\end{tabular}

Figure 3. Community structure for the NCS-R network. Communities 1 and 2 are highlighted with yellow and blue shading, respectively. The solid lines indicate three of the (maximum) 7cliques that were found. The dashed lines show a 4-clique that brought symptom 10 into Community 1. 


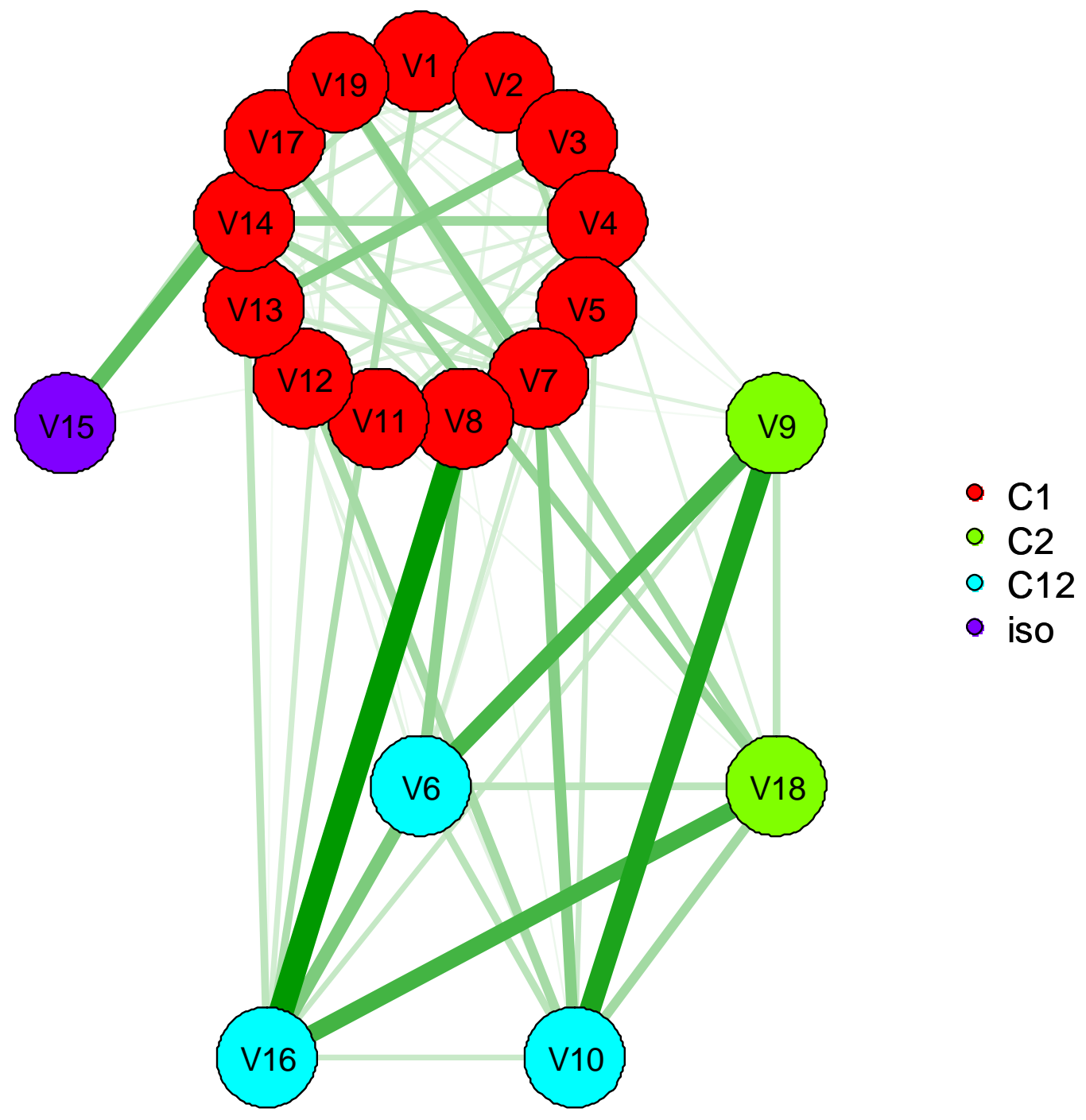

Figure 4. Network graph for the two-community solution in Table 4. Symptoms in C1 and C2, are, respectively, members of communities 1 and 2 only. Symptoms in C12 are members of both communities 1 and 2. Symptom 15 is an isolate that is not assigned a community membership. 


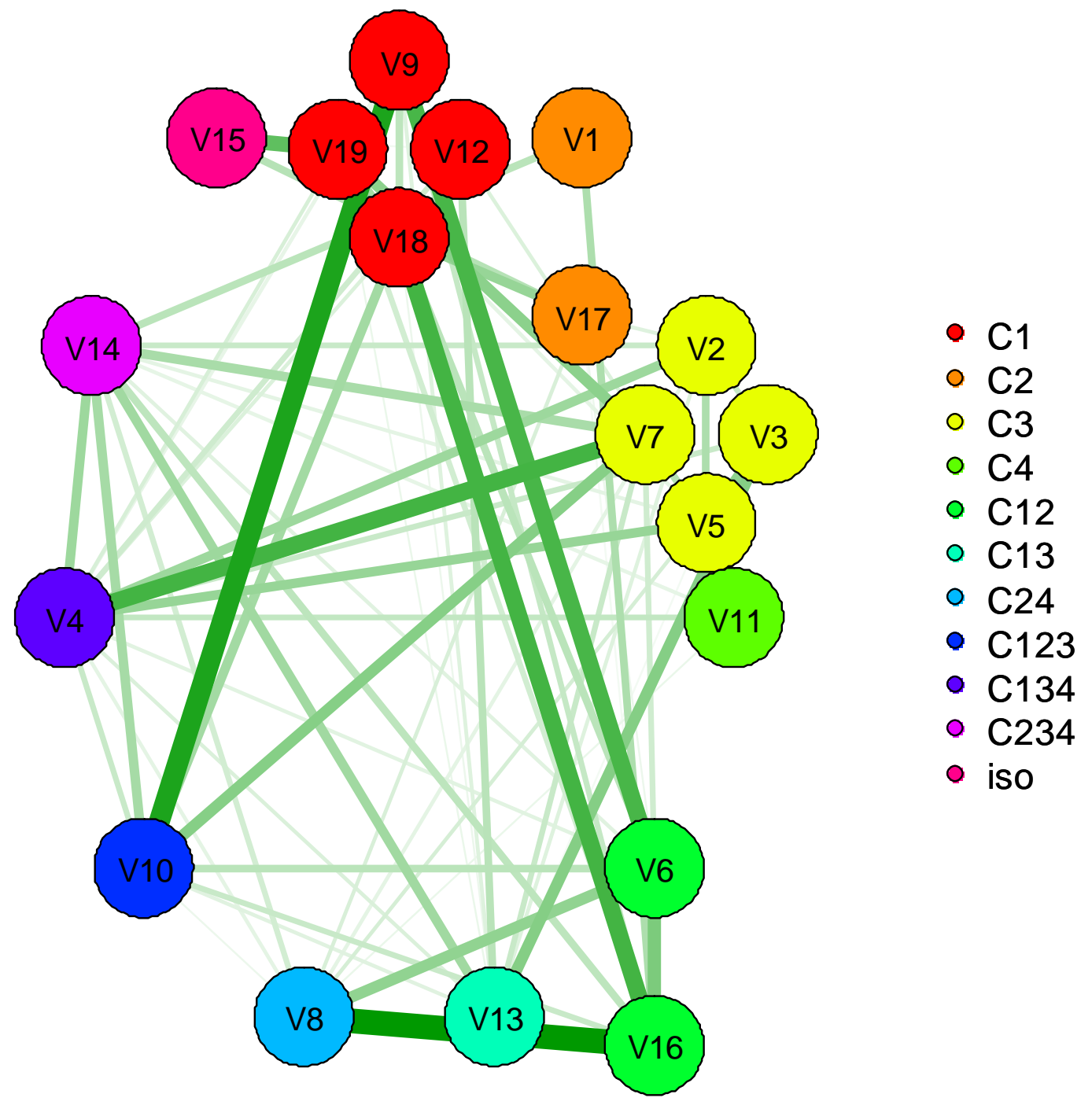

Figure 5. Network graph for the four-community solution in Table 4. Symptoms in C1, C2, C3, and $\mathrm{C} 4$ are, respectively, members of communities 1, 2, 3 and 4 only. Symptoms in C12 are members of both communities 1 and 2, symptoms in C123 are members of communities 1, 2, and 3, etc. Symptom 15 is an isolate that is not assigned a community membership. 